Groups Geom. Dyn. 9 (2015), 1185-1229

DOI $10.4171 / \mathrm{GGD} / 338$
Groups, Geometry, and Dynamics

(C) European Mathematical Society

\title{
The irreducible components of the moduli space of dihedral covers of algebraic curves
}

\author{
Fabrizio Catanese, Michael Lönne, and Fabio Perroni ${ }^{1}$
}

\begin{abstract}
The main purpose of this paper is to introduce a new invariant for the action of a finite group $G$ on a compact complex curve of genus $g$. With the aid of this invariant we achieve the classification of the components of the locus (in the moduli space) of curves admitting an effective action by the dihedral group $D_{n}$. This invariant has later been used in [11] where the results of Livingston [29] and of Dunfield and Thurston [17] have been extended to the ramified case.
\end{abstract}

Mathematics Subject Classification (2010). 14H37, 14H30, 14H15, 57M12, 57M60, $20 \mathrm{~J} 99$.

Keywords. Algebraic curves, topological type of coverings, monodromy, Hurwitz spaces, Hurwitz vectors, dihedral group, homological invariant, moduli space of curves, curves with automorphisms, mapping class group

\section{Contents}

1 Introduction . . . . . . . . . . . . . . . . . 1186

2 Moduli spaces of $G$-covers . . . . . . . . . . . . . . . . . . . . . . . 1189

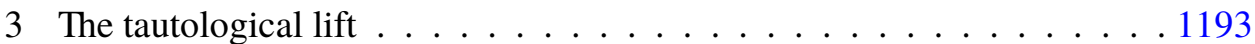

4 Computation of $H_{2, \Gamma}\left(D_{n}\right)$. . . . . . . . . . . . . . . . . . . 1200

5 The injectivity of $\hat{\varepsilon}$ when $G=D_{n} \ldots \ldots$. . . . . . . . . . . . . . . . . . . .

A Automorphisms of surface-groups . . . . . . . . . . . . . 1213

B Loci and topological types . . . . . . . . . . . . . 1218

References . . . . . . . . . . . . . . . . 1227

\footnotetext{
${ }^{1}$ The present work took place in the realm of the DFG Forschergruppe 790 'Classification of algebraic surfaces and compact complex manifolds.'
} 


\section{Introduction}

We study moduli spaces of curves that admit an effective action by a given finite group $G$. These moduli spaces can be seen as closed algebraic subsets $M_{g}(G)$ of $M_{g}$, the moduli space of smooth curves of genus $g>1$. We are mainly interested in understanding which are the irreducible components of $M_{g}(G)$.

To a curve $C$ of genus $g$ with an action by $G$, we can associate several discrete invariants that are constant under deformation.

On one hand, the topological type of the $G$-action is a homomorphism $\rho: G \rightarrow$ Map $_{g}$ well-defined up to inner conjugation induced by different choices of an isomorphism $\operatorname{Map}(C) \cong \operatorname{Map}_{g}$, see Section 2 .

It turns out that the locus $M_{g, \rho}(G)$ of curves admitting a $G$-action of topological type $\rho$ is a closed irreducible subset of $M_{g}$, (see Theorem 2.3).

On the other hand the action of $G$ on $C$ gives rise to a morphism $p: C \rightarrow C / G=: C^{\prime}$, a $G$-cover, and the geometry of $p$ encodes several numerical invariants that are constant on $M_{g, \rho}(G)$ : the genus $g^{\prime}$ of $C^{\prime}$, the number $d$ of branch points $y_{1}, \ldots, y_{d} \in C^{\prime}$ and the orders $m_{1} \leq \cdots \leq m_{d}$ of the local monodromies. These numbers $g^{\prime}, d, m_{1} \leq \cdots \leq m_{d}$ form the primary numerical type.

A second numerical invariant is obtained from the monodromy $\mu: \pi_{1}\left(C^{\prime} \backslash\left\{y_{1}, \ldots, y_{d}\right\}\right) \rightarrow G$ of the restriction of $p$ to $p^{-1}\left(C^{\prime} \backslash\left\{y_{1}, \ldots, y_{d}\right\}\right)$, and is called the $v$-type or Nielsen function. It is a class function $v$ which, for each conjugacy class $\mathcal{C}$ in $G$, counts the number of local monodromies which belong to $\mathcal{C}$.

Observe that the irreducible closed algebraic sets $M_{g, \rho}(G)$ depend only upon what we call the 'unmarked topological type', which is defined as the conjugacy class of the subgroup $\rho(G)$ inside $\operatorname{Map}_{g}$.

The following observation is immediate by Riemann's existence theorem and the irreducibility of the moduli space $M_{g^{\prime}, d}$ of $d$-pointed curves of genus $g^{\prime}$. Given $g^{\prime}$ and $d$, the unmarked topological types whose primary numerical type is of the form $g^{\prime}, d, m_{1}, \ldots, m_{d}$ are in bijection with the quotient of the set of the corresponding monodromies $\mu$ modulo the actions by $\operatorname{Aut}(G)$ and by $\operatorname{Map}\left(g^{\prime}, d\right)$. Here $\operatorname{Map}\left(g^{\prime}, d\right)$ is the full mapping class group of genus $g^{\prime}$ and $d$ unordered points. Thus a first step toward the general problem consists in finding a fine invariant that distinguishes these orbits.

In this paper we introduce a new invariant $\hat{\varepsilon}$ for $G$-actions on smooth curves.

In the case where $G$ is the dihedral group $D_{n}$ of order $2 n$, we show that $\hat{\varepsilon}$ distinguishes the different unmarked topological types, and therefore $\hat{\varepsilon}$ is a fine invariant in the dihedral case. 
Our invariant includes and extends two well known invariants that have been studied in the literature: the $v$-type (or Nielsen type) of the cover (also called shape in [21], cf. Definition 3.9) and the class in the second homology group $H_{2}(G / H, \mathbb{Z}$ ) (modulo the action of $\operatorname{Aut}(G / H)$ ) corresponding to the unramified cover $p^{\prime}: C / H \rightarrow C^{\prime}$, where $H$ is the minimal normal subgroup of $G$ generated by the local monodromies.

These invariants, which refine the primary numerical type, provide a fine invariant under some restrictions, for instance when $G$ is abelian or when $G$ acts freely and is the semi-direct product of two finite cyclic groups (as it follows by combining results from [7], [9], [18], and [19]). However, in general, they are not enough to distinguish unmarked topological types, as one can see already for non-free $D_{n}$-actions (see Lemma 5.9).

The construction of $\hat{\varepsilon}$ is similar in spirit to the procedure that, using Hopf's theorem, associates an element in $H_{2}(G, \mathbb{Z})$ to any free $G$-action on a smooth curve. So much cannot be achieved in the 'branched' case of a non-free action. In this case we are only able to associate to two given actions with the same $v$-type, an invariant in a quotient group of $H_{2}(G, \mathbb{Z})$ which is the 'difference' of the respective $\hat{\varepsilon}$ - invariants. Here is the way we do it. For any finite group $G$, let $F$ be the free group generated by the elements of $G$ and let $R \unlhd F$ be the subgroup of relations, that is $G=F / R$. For any $\Gamma \subset G$, union of non trivial conjugacy classes, let $G_{\Gamma}$ be the quotient group of $F$ by the minimal normal subgroup generated by $[F, R]$ and by the elements $\hat{a} \hat{b}^{-1} \hat{b}^{-1} \in F$, for any $a, c \in \Gamma$, $b \in G$, such that $b^{-1} a b=c$. Here we denote by $\hat{g} \in F$ the generator corresponding to $g \in G$. To a given $G$-cover $p: C \rightarrow C^{\prime}$ we associate the set $\Gamma$ of local monodromies, i.e., of elements which i) stabilize some point $x$ of $C$ and ii) act on the tangent space at $x$ by a rotation of angle $\frac{2 \pi}{m}$ where $m$ is the order of the stabilizer at $x$. Upon the choice of a geometric basis for the fundamental group of the complement $C^{\prime} \backslash\left\{y_{1}, \ldots, y_{d}\right\}$ of the branch set, our cover is given by an element $v=\left(c_{1}, \ldots, c_{d} ; a_{1}, b_{1}, \ldots, a_{g^{\prime}}, b_{g^{\prime}}\right) \in G^{d+2 g^{\prime}}$ satisfying certain conditions (a Hurwitz generating system), where the first entries correspond to the local monodromies. Thereby $\Gamma=\Gamma_{v}$ is the union of the conjugacy classes of the $c_{i}$ 's. The tautological lift $\hat{v}$ of $v$ is $\left(\widehat{c_{1}}, \ldots, \widehat{c_{d}} ; \widehat{a_{1}}, \widehat{b_{1}}, \ldots, \widehat{a_{g^{\prime}}}, \widehat{b_{g^{\prime}}}\right)$. Finally, define $\varepsilon(v)$ as the class in $G_{\Gamma}$ of

$$
\prod_{1}^{d} \widehat{c_{j}} \cdot \prod_{1}^{g^{\prime}}\left[\widehat{a_{i}}, \widehat{b_{i}}\right] .
$$

It turns out that the image of $\varepsilon(v)$ in $G_{\Gamma}$ is invariant under the action of $\operatorname{Map}\left(g^{\prime}, d\right)$, as shown in Proposition 3.6. Moreover the $v$-type of $v$ can be deduced from $\varepsilon(v)$, as it is essentially the image of $\varepsilon(v)$ in the abelianized group $G_{\Gamma}^{\text {ab }}$ (see the Remark after Defininition 3.9). 
In order to take into account also the automorphism group $\operatorname{Aut}(G)$, we define

$$
G^{\cup}:=\coprod_{\Gamma} G_{\Gamma},
$$

the disjoint union of all the $G_{\Gamma}$ 's. Now, the group $\operatorname{Aut}(G)$ acts on $G^{\cup}$ and we get a map

$$
\hat{\varepsilon}:\left(\operatorname{HS}\left(G ; g^{\prime}, d\right) / \operatorname{Aut}(G)\right) / \operatorname{Map}\left(g^{\prime}, d\right) \longrightarrow\left(G^{\cup}\right) / \operatorname{Aut}(G),
$$

which is induced by $v \mapsto \varepsilon(v)$. Here we denote by $\operatorname{HS}\left(G ; g^{\prime}, d\right)$ the set of all Hurwitz generating systems of length $d+2 g^{\prime}$.

Finally, we prove that the map $\hat{\varepsilon}$ is injective in the case $G=D_{n}$ and we determine the image of $\hat{\varepsilon}$, (Theorem 5.1), thus the invariant $\hat{\varepsilon}$ is a fine invariant for $D_{n}$-actions. This completes the classification of the unmarked topological types for $G=D_{n}$, begun in [10].

We finally show how this classification entails the classification of the irreducible components of the loci $M_{g}\left(D_{n}\right)$.

When $g^{\prime}=0$ our $G_{\Gamma}$ is related to the group $\widehat{G}$ defined in [21] (Appendix), where the authors give a proof of a theorem by Conway and Parker. Roughly speaking the theorem says that: if the Schur multiplier $M(G)$ (which is isomorphic to $H_{2}(G, \mathbb{Z})$ ) is generated by commutators, then the $v$-type is a fine stable invariant, when $g^{\prime}=0$.

Results of this kind, when $g^{\prime}>0$ but for free $G$-actions and any finite group $G$, have been proved in [29] and [17]. This time the fine stable invariant lives in $H_{2}(G, \mathbb{Z}) / \operatorname{Aut}(G)$.

The natural question whether our $\hat{\varepsilon}$-invariant is a fine stable invariant for any finite group $G$ and any effective $G$-action on compact curves has been solved in [11] for genus stabilisation.

The structure of the paper is the following. In Section 2 we introduce the moduli spaces $M_{g}(G)$ and the subsets $M_{g, \rho}(G)$. Using Riemann's existence theorem, we reduce the problem of the determination of the loci $M_{g, \rho}(G)$ to a combinatorial one. This leads to the concept of topological type and of Hurwitz generating system. In Section 3 we define the map $\hat{\varepsilon}$, the groups $H_{2, \Gamma}(G)$ and we prove some properties. The object of Section 4 is the computation of $H_{2, \Gamma}\left(D_{n}\right)$. These results are all used in Section 5 where we prove the injectivity of $\hat{\varepsilon}$ when $G=D_{n}$. In Appendix A we collect some results about mapping class groups and their action on fundamental groups. We use these results in the proof of Theorem 5.1.

Appendix B describes the case (see especially Theorem B.8) where two irreducible loci $M_{g, \rho}\left(D_{n}\right)$ coincide. 


\section{Moduli spaces of $G$-covers}

Throughout this section, $g$ is an integer, $g>1$. The moduli space of curves of genus $g$ is denoted by $M_{g}$. For any finite group $G, M_{g}(G)$ is the locus of $[C] \in M_{g}$ such that there exists an effective action of $G$ on $C$. For any $[C] \in M_{g}(G)$, the quotient morphism $p: C \rightarrow C / G=C^{\prime}$ is a Galois cover with group $G$, a $G$-cover, well defined up to isomorphisms.

Riemann's existence theorem allows us to use combinatorial methods to study $G$-covers, since $p$ determines and is determined by its restriction to $C^{\prime} \backslash \mathcal{B}$, where $\mathcal{B}=\left\{y_{1}, \ldots, y_{d}\right\} \subset C^{\prime}$ is the branch locus of $p$.

Fix a base point $y_{0} \in C^{\prime} \backslash \mathcal{B}$ and a point $x_{0} \in p^{-1}\left(y_{0}\right)$. Monodromy gives a surjective group-homomorphism

$$
\mu: \pi_{1}\left(C^{\prime} \backslash \mathcal{B}, y_{0}\right) \longrightarrow G
$$

that characterizes $p$ up to isomorphism.

Let us recall that a geometric basis of $\pi_{1}\left(C^{\prime} \backslash \mathcal{B}, y_{0}\right)$ consists of simple nonintersecting geometric loops based at $y_{0}$

$$
\gamma_{1}, \ldots, \gamma_{d}, \alpha_{1}, \beta_{1}, \ldots, \alpha_{g^{\prime}}, \beta_{g^{\prime}}
$$

such that we get the presentation

$$
\pi_{1}\left(C^{\prime} \backslash \mathcal{B}, y_{0}\right)=\left\langle\gamma_{1}, \ldots, \gamma_{d} ; \alpha_{1}, \beta_{1}, \ldots, \alpha_{g^{\prime}}, \beta_{g^{\prime}} \mid \prod_{1}^{d} \gamma_{j} \cdot \prod_{1}^{g^{\prime}}\left[\alpha_{i}, \beta_{i}\right]=1\right\rangle .
$$

Varying a covering in a flat family with connected base, there are some numerical invariants which remain unchanged, the first ones being the respective genera $g, g^{\prime}$ of the curves $C, C^{\prime}$, which are related by the Hurwitz formula:

$$
2(g-1)=|G|\left[2\left(g^{\prime}-1\right)+\sum_{i}\left(1-\frac{1}{m_{i}}\right)\right], \quad m_{i}:=\operatorname{ord}\left(\mu\left(\gamma_{i}\right)\right) .
$$

Observe moreover that a different choice of the geometric basis changes the generators $\gamma_{i}$, but does not change their conjugacy classes (up to permutation), hence another numerical invariant is provided by the number of elements $\mu\left(\gamma_{i}\right)$ which belong to a fixed conjugacy class in the group $G$.

We formalize these invariants through the following definition. 
Definition 2.1. Let $G$ be a finite group, and $g^{\prime}, d \in \mathbb{N}$. A $\left(g^{\prime}, d\right)$-Hurwitz vector in $G$ is an element $v \in G^{d+2 g^{\prime}}$, the Cartesian product of $G\left(d+2 g^{\prime}\right)$-times. A $\left(g^{\prime}, d\right)$-Hurwitz vector in $G$ will also be denoted by

$$
v=\left(c_{1}, \ldots, c_{d} ; a_{1}, b_{1}, \ldots, a_{g^{\prime}}, b_{g^{\prime}}\right) .
$$

For any $i \in\left\{1, \ldots, d+2 g^{\prime}\right\}$, the $i$-th component $v_{i}$ of $v$ is defined as usual. The evaluation of $v$ is the element

$$
\operatorname{ev}(v)=\prod_{1}^{d} c_{j} \cdot \prod_{1}^{g^{\prime}}\left[a_{i}, b_{i}\right] \in G .
$$

A Hurwitz generating system of length $d+2 g^{\prime}$ in $G$ is a $\left(g^{\prime}, d\right)$-Hurwitz vector $v$ in $G$ such that the following conditions hold:

(i) $c_{i} \neq 1$ for all $i$;

(ii) $G$ is generated by the components $v_{i}$ of $v$;

(iii) $\operatorname{ev}(v)=1$

We denote by $\operatorname{HS}\left(G ; g^{\prime}, d\right) \subset G^{d+2 g^{\prime}}$ the set of all Hurwitz generating systems in $G$ of length $d+2 g^{\prime}$.

Definition 2.2. The condition $\operatorname{ev}(v)=\prod_{1}^{d} c_{j} \cdot \prod_{1}^{g^{\prime}}\left[a_{i}, b_{i}\right]=1$ immediately implies that the product $\prod_{1}^{d} c_{j}$ has trivial image in the abelianization $G^{\mathrm{ab}}$ of $G$. Observe that the image of $c_{j}$ inside $G^{\mathrm{ab}}$ only depends on the conjugacy class $\mathcal{C}_{j}$ of $c_{j}$.

Denote by [C], for each conjugacy class $\mathcal{C}$ in $G$, its image inside $G^{\text {ab }}$.

One defines the Nielsen class function of $v$ as the function which, on each conjugacy class $\mathcal{C}$ in $G$, takes the value

$$
v(v)(\mathcal{C}):=\left|\left\{j \mid c_{j} \in \mathcal{C}\right\}\right| .
$$

We shall say that a class function $v:\{\mathcal{C}\} \rightarrow \mathbb{N}$ is admissible if it satisfies

$$
\sum_{\mathcal{C}} v(\mathcal{C})[\mathcal{C}]=0 \in G^{\mathrm{ab}} .
$$

Notice that, once we fix a base point $y_{0} \in C^{\prime} \backslash \mathcal{B}$ and a geometric basis of $\pi_{1}\left(C^{\prime} \backslash \mathcal{B}, y_{0}\right)$, there is a one-to-one correspondence between the set of Hurwitz generating systems of length $d+2 g^{\prime}$ in $G$ and the set of monodromies $\mu$ as in (1). 
Topological type. We recall a result contained in [7], see also [8].

Define the orbifold fundamental group $\pi_{1}^{\text {orb }}\left(C^{\prime} \backslash \mathcal{B}, y_{0} ; m_{1}, \ldots m_{d}\right)$ as the quotient of $\pi_{1}\left(C^{\prime} \backslash \mathcal{B}, y_{0}\right)$ by the minimal normal subgroup generated by the elements $\left(\gamma_{i}\right)^{m_{i}}$. If $p: C \rightarrow C^{\prime}$ is a $G$-covering as above, then its restriction to $C^{\prime} \backslash \mathcal{B}$ is a regular topological cover with short exact homotopy sequence

$$
1 \longrightarrow \pi_{1}\left(C \backslash p^{-1} \mathcal{B}, x_{0}\right) \longrightarrow \pi_{1}\left(C^{\prime} \backslash \mathcal{B}, y_{0}\right) \longrightarrow G \longrightarrow 1
$$

The corresponding exact sequence in orbifold covering theory is

$$
1 \longrightarrow \pi_{1}\left(C, x_{0}\right) \longrightarrow \pi_{1}^{\mathrm{orb}}\left(C^{\prime} \backslash \mathcal{B}, y_{0} ; m_{1}, \ldots m_{d}\right) \longrightarrow G \longrightarrow 1
$$

which is completely determined by the monodromy. In turn the exact sequence determines, via conjugation, a homomorphism

$$
\rho: G \longrightarrow \mathrm{Out}^{+}\left(\pi_{1}\left(C, x_{0}\right)\right)=\operatorname{Map}(C):=\operatorname{Diff}^{+}(C) / \operatorname{Diff}^{0}(C)
$$

which is fully equivalent to the topological action of $G$ on $C$.

Here the superscript ${ }^{+}$denotes orientation-preserving, and the superscript ${ }^{0}$ denotes 'isotopic to the identity'. The image is contained in the index two subgroup of outer automorphisms corresponding to orientation preserving mapping classes under the Dehn-Nielsen-Baer theorem, cf. [20, Theorem 8.1].

By Lemma 4.12 of [7], all the curves $C$ of a fixed genus $g$ which admit a given topological action $\rho$ of the group $G$ are parametrized by a connected complex manifold; arguing as in Theorem 2.4 of [9] we get

Theorem 2.3. The triples $(C, G, \rho)$ where $C$ is a complex projective curve of genus $g \geq 2$, and $G$ is a finite group acting effectively on $C$ with a topological action of type $\rho$ are parametrized by a connected complex manifold $\mathcal{T}_{g ; G, \rho}$ of dimension $3\left(g^{\prime}-1\right)+d$, where $g^{\prime}$ is the genus of $C^{\prime}=C / G$, and $d$ is the cardinality of the branch locus $\mathcal{B}$.

The image $M_{g, \rho}(G)$ of $\mathcal{T}_{g ; G, \rho}$ inside the moduli space $M_{g}$ is an irreducible closed subset of the same dimension $3\left(g^{\prime}-1\right)+d$.

Obviously, composing $\rho$ with an automorphism $\varphi \in \operatorname{Aut}(G)$, i.e. replacing $\rho$ with $\rho \circ \varphi$, does not change the subgroup $\rho(G) \subset \operatorname{Map}(C)$. In particular, $M_{g, \rho}(G)=M_{g, \rho \circ \varphi}(G)$, and similarly $\mathcal{T}_{g ; G, \rho}=\mathcal{T}_{g ; G, \rho \circ \varphi}$. 
Notice that $M_{g}(G)=\bigcup_{\rho} M_{g, \rho}(G)$, hence the components of $M_{g}(G)$ are in one-to-one correspondence with a subset of the different unmarked topological types. So, the next question which the above result motivates is: when do two monodromies $\mu_{1}, \mu_{2}: \pi_{1}\left(C^{\prime} \backslash \mathcal{B}, y_{0}\right) \rightarrow G$ have the same unmarked topological type?

The answer is theoretically easy: the two covering spaces have the same unmarked topological type if and only if they are homeomorphic, hence if and only if $\mu_{1}$ and $\mu_{2}$ differ by:

- an automorphism of $G$;

- and a different choice of a geometric basis. This is realized by the action of a mapping class in

$$
\operatorname{Map}\left(g^{\prime}, d\right):=\frac{\operatorname{Diff}^{+}\left(C^{\prime}, \mathcal{B}\right)}{\operatorname{Diff}^{0}\left(C^{\prime}, \mathcal{B}\right)}
$$

To reformulate these conditions in terms of Hurwitz generating systems, notice that $\operatorname{Aut}(G)$ acts on $\operatorname{HS}\left(G ; g^{\prime}, d\right)$ componentwise, and $\operatorname{Map}\left(g^{\prime}, d\right)$ acts on $\operatorname{HS}\left(G ; g^{\prime}, d\right) / \operatorname{Aut}(G)$. The latter action is given by the group homomorphism $\operatorname{Map}\left(g^{\prime}, d\right) \rightarrow \operatorname{Out}\left(\pi_{1}\left(C^{\prime} \backslash \mathcal{B}, y_{0}\right)\right)$ and the identification between monodromies $\mu$ and Hurwitz generating systems. Theorem 2.3 then implies that there is a bijection between the set of unmarked topological types $[\rho]$ with $g^{\prime}$ and $d$ fixed, and the following orbit space

$$
\{[\rho]\} \longleftrightarrow\left(\operatorname{HS}\left(G ; g^{\prime}, d\right) / \operatorname{Aut}(G)\right) / \operatorname{Map}\left(g^{\prime}, d\right) .
$$

In the next sections, we will also use the action of the unpermuted mapping class group

$$
\operatorname{Map}^{u}\left(g^{\prime}, d+1\right):=\operatorname{Map}^{u}\left(C^{\prime}, \mathcal{B} \cup\left\{y_{0}\right\}\right)
$$

on $\operatorname{HS}\left(G ; g^{\prime}, d\right)$, where $\operatorname{Map}^{u}\left(g^{\prime}, d+1\right)$ consists of diffeomorphisms in $\operatorname{Diff}^{+}\left(C^{\prime}\right)$ which are the identity on $\mathcal{B} \cup\left\{y_{0}\right\}$, modulo isotopy. For any $v_{1}, v_{2} \in \operatorname{HS}\left(G ; g^{\prime}, d\right)$, we write $v_{1} \sim v_{2}$ when they are in the same $\operatorname{Map}^{u}\left(g^{\prime}, d+1\right)$-orbit. While $v_{1} \approx v_{2}$ means that they represent the same class in $\left(\operatorname{HS}\left(G ; g^{\prime}, d\right) / \operatorname{Aut}(G)\right) / \operatorname{Map}\left(g^{\prime}, d\right)$. Clearly $v_{1} \sim v_{2}$ implies $v_{1} \approx v_{2}$.

The mapping class group $\operatorname{Map}\left(g^{\prime}, d\right)$ acts on $\operatorname{HS}\left(G ; g^{\prime}, d\right)$ only up to conjugation, but, since we are interested in classifying Hurwitz generating systems up to $\operatorname{Aut}(G)$, we will also use the notation $\varphi \cdot v$, meaning $\varphi \cdot[v]$, with $\varphi \in \operatorname{Map}\left(g^{\prime}, d\right)$ and $[v] \in \operatorname{HS}\left(G ; g^{\prime}, d\right) / \operatorname{Aut}(G)$. 


\section{The tautological lift}

In this section we give the construction of our invariant in several steps. Having defined a suitable group $G_{\Gamma}$, for any $\Gamma \subset G$ union of non-trivial conjugacy classes, we go on to a map $\varepsilon$, which associates to each Hurwitz vector $v$ (with $c_{i} \in \Gamma$ ) an element $\varepsilon(v) \in G_{\Gamma}$. Any automorphism $f \in \operatorname{Aut}(G)$ induces an isomorphism

$$
f_{\Gamma}: G_{\Gamma} \rightarrow G_{f(\Gamma)}
$$

hence $\operatorname{Aut}(G)$ acts on the disjoint union

$$
G^{\cup}=\coprod_{\Gamma} G_{\Gamma} .
$$

We show two key properties of $\varepsilon$ :

- it is $\operatorname{Aut}(G)$-equivariant (Lemma 3.5), hence it descends to a map

$$
\tilde{\varepsilon}: \operatorname{HS}\left(G ; g^{\prime}, d\right) / \operatorname{Aut}(G) \longrightarrow G^{\cup} / \operatorname{Aut}(G) ;
$$

- $\tilde{\varepsilon}$ is constant on the orbits of the mapping class group $\operatorname{Map}\left(g^{\prime}, d\right)$ (Proposition 3.6).

Therefore $\varepsilon$ descends to our invariant $\hat{\varepsilon}$ which is formalized as the map

$$
\hat{\varepsilon}:\left(\operatorname{HS}\left(G ; g^{\prime}, d\right) / \operatorname{Aut}(G)\right) / \operatorname{Map}\left(g^{\prime}, d\right) \longrightarrow G^{\cup} / \operatorname{Aut}(G), \quad \text { for all } g^{\prime}, d,
$$

induced by $\varepsilon$. We conclude the section with the study of general properties of the invariant that are relevant to this work.

Since our construction is inspired by Hopf's description of the second homology group $H_{2}(G, \mathbb{Z})$ [24], we begin by recalling this. For a finite group $G$, fix a presentation of $G$ :

$$
1 \longrightarrow R \longrightarrow F \longrightarrow G \longrightarrow 1,
$$

where $F$ is a free group. Then there is a group isomorphism (cf. [5]):

$$
H_{2}(G, \mathbb{Z}) \cong \frac{R \cap[F, F]}{[F, R]} .
$$

If $v=\left(a_{1}, b_{1}, \ldots, a_{g^{\prime}}, b_{g^{\prime}}\right) \in G^{2 g^{\prime}}$ satisfies $\prod_{1}^{g^{\prime}}\left[a_{i}, b_{i}\right]=1$, then we can associate a class in $H_{2}(G, \mathbb{Z})$ in the following way: choose liftings $\widehat{a_{i}}, \widehat{b_{i}} \in F$ of $a_{i}, b_{i}$, then $\prod_{1}^{g^{\prime}}\left[\widehat{a_{i}}, \widehat{b_{i}}\right] \in R \cap[F, F]$ and its class in $\frac{R \cap[F, F]}{[F, R]}$ gives an element of $H_{2}(G, \mathbb{Z})$, according to (4). Clearly, this element does not depend on the choices of lifts, moreover it is constant on the equivalence class of $v$ modulo simultaneous conjugation and under the action of the mapping class group, thus giving a topological invariant of $v$. 
The topological meaning of this invariant is the following. If the $G$-action on $C$ is free, the covering $p: C \rightarrow C^{\prime}$ is étale, and hence it corresponds to a continuous function $B p: C^{\prime} \rightarrow B G$, up to homotopy. Here $B G$ is the classifying space of $G$. The topological invariant is simply the image $B p_{*}\left(\left[C^{\prime}\right]\right) \in H_{2}(B G, \mathbb{Z})=$ $H_{2}(G, \mathbb{Z})$ of the fundamental class $\left[C^{\prime}\right] \in H_{2}\left(C^{\prime}, \mathbb{Z}\right)$ of $C^{\prime}$ under the homomorphism $B p_{*}: H_{2}\left(C^{\prime}, \mathbb{Z}\right) \rightarrow H_{2}(B G, \mathbb{Z})$ induced by $B p$. Now, if we view $C^{\prime}$ as an Eilenberg-Mac Lane space $K\left(\pi_{1}\left(C^{\prime}\right), 1\right)$, then the fundamental class $\left[C^{\prime}\right]$ is given by

$$
\prod_{1}^{g^{\prime}}\left[\widehat{\alpha_{i}}, \widehat{\beta_{i}}\right] \in H_{2}\left(\pi_{1}\left(C^{\prime}\right), \mathbb{Z}\right),
$$

where as usual $\alpha_{1}, \beta_{1}, \ldots, \alpha_{g^{\prime}}, \beta_{g^{\prime}}$ is a geometric basis of $\pi_{1}\left(C^{\prime}\right)$ and $\widehat{\alpha_{i}}, \widehat{\beta_{i}}$ are liftings to the free group of a presentation of $\pi_{1}\left(C^{\prime}\right)$. So,

$$
B p_{*}\left(\left[C^{\prime}\right]\right)=\prod_{1}^{g^{\prime}}\left[\widehat{a_{i}}, \widehat{b_{i}}\right] \in H_{2}(G, \mathbb{Z}),
$$

where $a_{i}=\mu\left(\alpha_{i}\right), b_{i}=\mu\left(\beta_{i}\right)$ and $\mu: \pi_{1}\left(C^{\prime}\right) \rightarrow G$ is the monodromy of $p: C \rightarrow C^{\prime}$.

From now on, $F=\langle\hat{g} \mid g \in G\rangle$ is the free group generated by the elements of $G$. Let $R \unlhd F$ be the minimal normal subgroup generated by the relations, that is $G=\frac{F}{R}$.

Definition 3.1. Let $G$ be a finite group and let $F, R$ be as above. For any union of non-trivial conjugacy classes $\Gamma \subset G$, define

$$
\left.R_{\Gamma}=\left\langle\left\langle[F, R], \hat{a} \hat{b} \hat{c}^{-1} \hat{b}^{-1}\right| \text { for all } a \in \Gamma, a b=b c\right\rangle\right\rangle
$$

and

$$
G_{\Gamma}=\frac{F}{R_{\Gamma}} .
$$

The map $\hat{a} \mapsto a$, for all $a \in G$, induces a group homomorphism $\alpha: G_{\Gamma} \rightarrow G$. Set $K_{\Gamma}=\operatorname{ker}(\alpha)$.

Lemma 3.2. With the notation as before, the following holds. $R_{\Gamma} \subset R$ and $K_{\Gamma}=\frac{R}{R_{\Gamma}}$. In particular $K_{\Gamma}$ is contained in the center of $G_{\Gamma}$ and the short exact sequence

$$
1 \longrightarrow \frac{R}{R_{\Gamma}} \longrightarrow G_{\Gamma} \stackrel{\alpha}{\longrightarrow} G \longrightarrow 1
$$

is a central extension. 
Proof. $[F, R] \subset R$ because $R$ is normal in $F$ and moreover $\hat{a} \hat{b}^{-1} \hat{b}^{-1} \in R$ for any $a, b, c \in G$ with $a b=b c$, therefore $R_{\Gamma} \subset R$. By the definition of $\alpha$ we have that $K_{\Gamma}=\frac{R}{R_{\Gamma}}$. Finally, $K_{\Gamma}$ is in the center of $G_{\Gamma}$ because $[F, R] \subset R_{\Gamma}$.

The morphism $\alpha: G_{\Gamma} \rightarrow G$ has a tautological section $G \rightarrow G_{\Gamma}, a \mapsto \hat{a}$. This map is not a group homomorphism in general, but every element $\xi \in G_{\Gamma}$ can be written as $\hat{g} z=z \hat{g}$, with $g=\alpha(\xi) \in G$ and $z \in K_{\Gamma}$. Here, by abuse of notation, $\hat{a}$ denotes also the class of $\hat{a} \in F$ in $G_{\Gamma}=F / R_{\Gamma}$.

Lemma 3.3. Let $\hat{a}, \xi \in G_{\Gamma}$. Suppose that $\hat{a}$ is conjugate to $\xi$ in $G_{\Gamma}$ and that $a \in \Gamma$. Then $\xi=\widehat{\alpha(\xi)}$.

Proof. Let $\hat{b} z$ be a conjugating element, that is $\hat{a} \hat{b} z=\hat{b} z \xi$. As $z \in K_{\Gamma}$, it commutes with any element, hence

$$
\hat{a} \hat{b}=\hat{b} \xi
$$

Now apply $\alpha$ and obtain: $a b=b \alpha(\xi)$. By assumption $a \in \Gamma$, hence by definition of $G_{\Gamma}$ we have that $\hat{a} \hat{b}=\hat{b} \widehat{\alpha(\xi)}$. Now using (5) we deduce $\xi=\widehat{\alpha(\xi)}$.

Definition 3.4. Given a $\left(g^{\prime}, d\right)$-Hurwitz vector

$$
v=\left(c_{1}, \ldots, c_{d} ; a_{1}, b_{1}, \ldots, a_{g^{\prime}}, b_{g^{\prime}}\right)
$$

in $G$, cf. Definition 2.1, its tautological lift $\hat{v}$ is the $\left(g^{\prime}, d\right)$-Hurwitz vector in $G_{\Gamma}$ defined by

$$
\hat{v}=\left(\widehat{c_{1}}, \ldots, \widehat{c_{d}} ; \widehat{a_{1}}, \widehat{b_{1}}, \ldots, \widehat{a_{g^{\prime}}}, \widehat{b_{g^{\prime}}}\right)
$$

where the factors are the tautological lifts of the factors of $v$.

Given a $\left(g^{\prime}, d\right)$-Hurwitz vector $v$ in $G$ with $c_{i} \neq 1$, for all $i$, we denote by $\Gamma_{v}$ the union of all conjugacy classes of $G$ containing at least one $c_{i}$.

For any $v$ as before, let

$$
\varepsilon(v)=\prod_{1}^{d} \widehat{c_{j}} \cdot \prod_{1}^{g^{\prime}}\left[\widehat{a_{i}}, \widehat{b_{i}}\right] \in G_{\Gamma_{v}},
$$

be the evaluation of the tautological lift $\hat{v}$ of $v$ in $G_{\Gamma_{v}}$, in analogy to Definition 2.1. 
Lemma 3.5. Let $G$ be any finite group, and let $\Gamma \subset G$ be any union of non trivial conjugacy classes. Then

i) any $f \in \operatorname{Aut}(G)$ induces an isomorphism $f_{\Gamma}: G_{\Gamma} \rightarrow G_{f(\Gamma)}$;

ii) $\varepsilon(f(v))=f_{\Gamma}(\varepsilon(v))$, for all $f \in \operatorname{Aut}(G)$ and for all $v$ a $\left(g^{\prime}, d\right)$-Hurwitz vector with $c_{i} \neq 1$, for all $i$, where $\Gamma=\Gamma_{v}$.

Proof. i) $f \in \operatorname{Aut}(G)$ lifts to an automorphism $\hat{f} \in \operatorname{Aut}(F)$ defined by

$$
\hat{f}: \hat{a} \longmapsto \widehat{f(a)} .
$$

We have $\hat{f}(R) \subset R$, and moreover

$$
\left.\left.\hat{f}\left(\hat{a} \hat{b}^{-1} \hat{b}^{-1}\right)=\widehat{f(a)} \widehat{f(b)} \widehat{f(c)}\right)^{-1} \widehat{f(b)}\right)^{-1},
$$

for any $a, b, c \in G$. If $a \in \Gamma$, then $f(a) \in f(\Gamma)$ and hence

$$
\widehat{f(a)} \widehat{f(b)} \widehat{f(c)}^{-1} \widehat{f(b)}-1 \in R_{f(\Gamma)} .
$$

ii)

$$
\begin{aligned}
\varepsilon(f(v)) & =\varepsilon\left(f\left(c_{1}\right), \ldots, f\left(c_{d}\right) ; f\left(a_{1}\right), \ldots, f\left(b_{g^{\prime}}\right)\right) \\
& =\prod_{1}^{d} \widehat{f\left(c_{i}\right)} \cdot \prod_{1}^{g^{\prime}}\left[\widehat{f\left(a_{j}\right)}, \widehat{f\left(b_{j}\right)}\right] \\
& =\prod_{1}^{d} \hat{f}\left(\widehat{c_{i}}\right) \cdot \prod_{1}^{g^{\prime}}\left[\hat{f}\left(\widehat{a_{j}}\right), \hat{f}\left(\widehat{b_{j}}\right)\right] \\
& =f_{\Gamma}(\varepsilon(v)) .
\end{aligned}
$$

Now, we define

$$
G^{\cup}:=\coprod_{\Gamma} G_{\Gamma},
$$

and regard $\varepsilon$ as a map $\varepsilon$ : $\operatorname{HS}\left(G ; g^{\prime}, d\right) \rightarrow G^{\cup}, v \mapsto \varepsilon(v) \in G_{\Gamma_{v}}$. Then the previous lemma means that $\varepsilon$ induces a map

$$
\tilde{\varepsilon}: \operatorname{HS}\left(G ; g^{\prime}, d\right) / \operatorname{Aut}(G) \longrightarrow\left(G^{\cup}\right) / \operatorname{Aut}(G) .
$$

We have the following

Proposition 3.6. For any $g^{\prime}, d \in \mathbb{N}, \tilde{\varepsilon}$ is $\operatorname{Map}\left(g^{\prime}, d\right)$-invariant, hence it induces a map

$$
\hat{\varepsilon}:\left(\operatorname{HS}\left(G ; g^{\prime}, d\right) / \operatorname{Aut}(G)\right) / \operatorname{Map}\left(g^{\prime}, d\right) \longrightarrow\left(G^{\cup}\right) / \operatorname{Aut}(G) .
$$


To prove this proposition we need some preliminary results.

Lemma 3.7. Let $\Gamma_{v}$ be associated to a $\left(g^{\prime}, d\right)$-Hurwitz vector $v$ as in Definition 3.4. If the Hurwitz vector $v^{\prime}$ is related to $v$ by an elementary braid move, then $\varepsilon(v)=\varepsilon\left(v^{\prime}\right)$.

Proof. It suffices to consider the case $g=0, d=2$ and the elementary braid move

$$
\sigma_{1}: v=\left(c_{1}, c_{2}\right) \longmapsto v^{\prime}=\left(c_{2}, c_{2}^{-1} c_{1} c_{2}\right) .
$$

In $G_{\Gamma_{v}}$ we have, thanks to $c_{1} \in \Gamma_{v}, c_{1} c_{2}=c_{2}\left(c_{2}^{-1} c_{1} c_{2}\right)$, and the relations of $G_{\Gamma_{v}}$ :

$$
\varepsilon(v)=\widehat{c_{1}} \widehat{c_{2}}=\widehat{c_{2}} \widehat{c_{2}^{-1} c_{1} c_{2}}=\varepsilon\left(v^{\prime}\right) \text {. }
$$

Lemma 3.8. If $\xi, \eta \in G_{\Gamma}$, then

$$
[\xi, \eta]=[\widehat{\alpha(\xi)}, \widehat{\alpha(\eta)}] .
$$

Proof. Write $\xi=\widehat{\alpha(\xi)} z$ and $\eta=\widehat{\alpha(\eta)} z^{\prime}$ with $z, z^{\prime}$ in $K_{\Gamma}$, hence central (see Lemma 3.2). Then the conclusion is immediate.

Proof of Proposition 3.6. Thanks to Lemma 3.7 it suffices to consider the invariance under the action of pure mapping classes, i.e. classes that do not permute the conjugacy classes associated to the local monodromies. Hence to prove the invariance of $\tilde{\varepsilon}$ under the action of $\operatorname{Map}\left(g^{\prime}, d\right)$ it suffices to prove the invariance of $\varepsilon$ under the action on $\operatorname{HS}\left(G ; g^{\prime}, d\right)$ by any $\varphi \in \operatorname{Map}^{u}\left(g^{\prime}, d+1\right)$, see (3).

Since $\varphi$ is a pure mapping class, the components of $v, \varphi \cdot v$ are conjugate to each other, $v_{i} \sim(\varphi \cdot v)_{i}$ for $i=1, \ldots, d$, and where $\sim$ denotes conjugation equivalence. For the components of $\hat{v}, \varphi \cdot \hat{v}$ the same is true, $\hat{v}_{i} \sim(\varphi \cdot \widehat{v})_{i}$ for $i=1, \ldots, d$. By Lemma 3.3 we thus have

$$
\left.(\hat{v})_{i} \sim(\varphi \cdot \hat{v})_{i} \Longrightarrow(\varphi \cdot \hat{v})_{i}=\overline{\alpha\left((\varphi \cdot \hat{v})_{i}\right.}\right) .
$$

Now notice that the homomorphism $\alpha$ of Definition 3.1 induces a map

$$
\alpha^{\left(d+2 g^{\prime}\right)}: \operatorname{HS}\left(G_{\Gamma} ; g^{\prime}, d\right) \longrightarrow \operatorname{HS}\left(G ; g^{\prime}, d\right),
$$

which is equivariant under the action of the mapping class group in the following sense: consider the factorizations as a map from the free group on $d+2 g^{\prime}$ generators to $G_{\Gamma}$, resp. $G$, and the mapping class group as a group of automorphisms 
of this free group. Then $\alpha^{\left(d+2 g^{\prime}\right)}$ is equivariant, since such automorphisms act by pre-composition. Hence by the equivariance of $\alpha^{\left(d+2 g^{\prime}\right)}$

$$
\alpha\left((\varphi \cdot \hat{v})_{i}\right)=(\varphi \cdot v)_{i}
$$

and therefore, for $i=1, \ldots, d$,

$$
(\varphi \cdot \hat{v})_{i}=\widehat{\alpha\left((\varphi \cdot \hat{v})_{i}\right)}=\widehat{(\varphi \cdot v)_{i}}=(\widehat{\varphi \cdot v})_{i} .
$$

By Lemma 3.8 we may change also the entries $(\varphi \cdot \hat{v})_{i}, i>d$ in the commutators to $\widehat{\alpha\left((\varphi \cdot \hat{v})_{i}\right)}=(\widehat{\varphi \cdot v})_{i}$ without changing the value of the commutators. Hence

$$
\operatorname{ev}(\varphi \cdot \hat{v})=\operatorname{ev}(\widehat{\varphi \cdot v})=\varepsilon(\varphi \cdot v) .
$$

By the invariance of the evaluation under the mapping class

$$
\varepsilon(v)=\operatorname{ev}(\hat{v})=\operatorname{ev}(\varphi \cdot \hat{v})=\varepsilon(\varphi \cdot v)
$$

and we have proved our claim.

Definition 3.9. Let $v \in \operatorname{HS}\left(G ; g^{\prime}, d\right)$ and let $v(v) \in \bigoplus_{\mathcal{C}} \mathbb{Z}\langle\mathcal{C}\rangle$ ( $\mathcal{C}$ runs over the set of conjugacy classes of $G$ ) be the vector whose $\mathcal{C}$-component is the number of $v_{j}, j \leq d$, which belong to $\mathcal{C}(v(v)$ is also called the shape of $v$ in [21]).

The map

$$
v: \operatorname{HS}\left(G ; g^{\prime}, d\right) \longrightarrow \bigoplus_{\mathcal{C}} \mathbb{Z}\langle\mathcal{C}\rangle
$$

obtained in this way induces a map

$$
\tilde{v}: \operatorname{HS}\left(G ; g^{\prime}, d\right) / \operatorname{Aut}(G) \longrightarrow\left(\bigoplus_{\mathcal{C}} \mathbb{Z}\langle\mathcal{C}\rangle\right) / \operatorname{Aut}(G)
$$

which is $\operatorname{Map}\left(g^{\prime}, d\right)$-invariant, therefore we get a map

$$
\hat{v}:\left(\operatorname{HS}\left(G ; g^{\prime}, d\right) / \operatorname{Aut}(G)\right) / \operatorname{Map}\left(g^{\prime}, d\right) \longrightarrow\left(\bigoplus_{\mathrm{e}} \mathbb{Z}\langle\mathcal{C}\rangle\right) / \operatorname{Aut}(G) .
$$

For any $v \in \operatorname{HS}\left(G ; g^{\prime}, d\right)$, we call $\hat{v}(v)$ the $v$-type of $v$.

Remark 3.10. Let $v \in \operatorname{HS}\left(G ; g^{\prime}, d\right)$ and let $\Gamma_{v} \subset G$ be the union of the conjugacy classes of $v_{j}, j \leq d$. The abelianization $G_{\Gamma_{v}}^{\text {ab }}$ of $G_{\Gamma_{v}}$ can be described as follows:

$$
G_{\Gamma_{v}}^{\mathrm{ab}} \cong \bigoplus_{\mathcal{C} \subset \Gamma_{v}} \mathbb{Z}\langle\mathcal{C}\rangle \oplus \underset{g \in G \backslash \Gamma_{v}}{\bigoplus_{v}} \mathbb{Z}\langle g\rangle,
$$

where $\mathcal{C}$ denotes a conjugacy class of $G$.

Observe that $v(v) \in \bigoplus_{\mathcal{C} \subset \Gamma_{v}} \mathbb{Z}\langle\mathcal{C}\rangle \subset G_{\Gamma_{v}}^{\text {ab }}$ coincides with the vector which is the image in $G_{\Gamma_{v}}^{\mathrm{ab}}$ of $\varepsilon(v) \in G_{\Gamma_{v}}$ under the natural homomorphism $G_{\Gamma_{v}} \rightarrow G_{\Gamma_{v}}^{\mathrm{ab}}$. 
Definition 3.11. Let $\Gamma \subset G$ be a union of non-trivial conjugacy classes of $G$. We define

$$
H_{2, \Gamma}(G)=\operatorname{ker}\left(G_{\Gamma} \longrightarrow G \times G_{\Gamma}^{\mathrm{ab}}\right),
$$

where $G_{\Gamma} \rightarrow G \times G_{\Gamma}^{\text {ab }}$ is the morphism with first component $\alpha$ (defined in Definition 3.1) and second component the natural morphism $G_{\Gamma} \rightarrow G_{\Gamma}^{\mathrm{ab}}$.

Notice that

$$
H_{2}(G, \mathbb{Z}) \cong \frac{R \cap[F, F]}{[F, R]} \cong \operatorname{ker}\left(\frac{F}{[F, R]} \rightarrow G \times G_{\emptyset}^{\mathrm{ab}}\right) .
$$

In particular, when $\Gamma=\emptyset, H_{2, \Gamma}(G) \cong H_{2}(G, \mathbb{Z})$.

The next result gives a precise relation between $H_{2}(G, \mathbb{Z})$ and $H_{2, \Gamma}(G)$.

Lemma 3.12. Let $G$ be a finite group and let $\Gamma \subset G$ be a union of nontrivial conjugacy classes. Write $G=\frac{F}{R}$ and $G_{\Gamma}=\frac{F}{R_{\Gamma}}$. Then, there is a short exact sequence

$$
1 \longrightarrow \frac{R_{\Gamma} \cap[F, F]}{[F, R]} \longrightarrow H_{2}(G, \mathbb{Z}) \longrightarrow H_{2, \Gamma}(G) \longrightarrow 1 .
$$

In particular $H_{2, \Gamma}(G)$ is abelian.

Proof. We first define the morphism $H_{2}(G, \mathbb{Z}) \rightarrow H_{2, \Gamma}(G)$.

By Hopf's Theorem we identify $H_{2}(G, \mathbb{Z})$ with $\frac{R \cap[F, F]}{[F, R]}$ (cf. [5]). On the other hand we have:

$$
H_{2, \Gamma}(G)=\operatorname{ker}\left(G_{\Gamma} \rightarrow G\right) \cap \operatorname{ker}\left(G_{\Gamma} \rightarrow G_{\Gamma}^{\mathrm{ab}}\right)=\frac{R}{R_{\Gamma}} \cap\left[G_{\Gamma}, G_{\Gamma}\right] .
$$

By Lemma 3.2, $R_{\Gamma} \subset R$. The homomorphism $R \cap[F, F] \rightarrow \frac{R}{R_{\Gamma}}, r \mapsto r R_{\Gamma}$, takes values in $H_{2, \Gamma}(G)$. Moreover it descends to a group homomorphism $H_{2}(G, \mathbb{Z}) \rightarrow H_{2, \Gamma}(G)$ because $[F, R] \subset R_{\Gamma}$.

To prove the surjectivity, let

$$
a R_{\Gamma} \in \frac{R}{R_{\Gamma}} \cap\left[G_{\Gamma}, G_{\Gamma}\right] .
$$

Since $a R_{\Gamma} \in\left[G_{\Gamma}, G_{\Gamma}\right]=\frac{[F, F] \cdot R_{\Gamma}}{R_{\Gamma}}$, we may assume $a \in[F, F]$. From $a R_{\Gamma} \in \frac{R}{R_{\Gamma}}$, we have $a R_{\Gamma}=r R_{\Gamma}$, for some $r \in R$. Since $R_{\Gamma} \subset R$, we deduce that $a \in R$ and so the surjectivity follows.

The kernel of the morphism so defined is $\frac{R_{\Gamma} \cap[F, F]}{[F, R]}$.

Since $H_{2}(G, \mathbb{Z})$ is abelian, so is $H_{2, \Gamma}(G)$. 
Proposition 3.13. Let $v_{1}, v_{2} \in \mathrm{HS}\left(G ; g^{\prime}, d\right)$ be two Hurwitz generating systems in $G$ with the same v-type. Then $\Gamma_{v_{1}}=\Gamma_{v_{2}}=: \Gamma$. Moreover, if $\operatorname{ev}\left(v_{1}\right)=\operatorname{ev}\left(v_{2}\right) \in G$, then the element

$$
\varepsilon\left(v_{1}\right)^{-1} \cdot \varepsilon\left(v_{2}\right) \in H_{2, \Gamma}(G)
$$

is invariant under the group $\widetilde{\operatorname{Map}}\left(g^{\prime}, d\right)$ of isotopy classes of orientation-preserving diffeomorphisms of the pair $\left(C^{\prime}, \mathcal{B}\right)$ that fix $y_{0}$. In particular,

(1) if $v_{1}$ and $v_{2}$ are equivalent then the element is trivial;

(2) if the element is non-trivial, then $v_{1}$ and $v_{2}$ are inequivalent.

\section{Computation of $H_{2}, \Gamma\left(D_{n}\right)$}

In this section we derive a complete description of $H_{2, \Gamma}(G)$ in the special case that $G$ is equal to the dihedral group

$$
\begin{aligned}
D_{n} & =\left\langle x, y \mid x^{n}=1, y^{2}=1, x y=y x^{-1}\right\rangle \\
& =\left\{x^{i} y^{j} \mid 0 \leq i<n, 0 \leq j<2\right\} .
\end{aligned}
$$

Proposition 4.1. Let $n \in \mathbb{N}, n \geq 3$. Then

(i) $H_{2}\left(D_{n}, \mathbb{Z}\right)$ is trivial if $n$ is odd and it is isomorphic to $\mathbb{Z} / 2 \mathbb{Z}$ if $n$ is even;

(ii) the natural action of $\operatorname{Aut}\left(D_{n}\right)$ on $H_{2}\left(D_{n}, \mathbb{Z}\right)$ is trivial.

Proof. (ii) This claim follows directly from (i) and from the fact that the neutral element of $H_{2}\left(D_{n}, \mathbb{Z}\right)$ is fixed by the action of $\operatorname{Aut}\left(D_{n}\right)$.

(i) Identify $D_{n}$ with the subgroup of $\mathrm{SO}(3)$ generated by

$$
x:=\left(\begin{array}{ccc}
\cos \frac{2 \pi}{n} & -\sin \frac{2 \pi}{n} & 0 \\
\sin \frac{2 \pi}{n} & \cos \frac{2 \pi}{n} & 0 \\
0 & 0 & 1
\end{array}\right) \quad \text { and } \quad y:=\left(\begin{array}{ccc}
-1 & 0 & 0 \\
0 & 1 & 0 \\
0 & 0 & -1
\end{array}\right) .
$$

Let $u: \mathrm{SU}(2) \rightarrow \mathrm{SO}(3)$ be the homomorphism $q \mapsto R_{q}$, where we identify $\mathrm{SU}(2)$ with the quaternions $q \in \mathbb{H}$ of norm $1, \mathbb{R}^{3}$ with $\operatorname{Im} \mathbb{H}$, and $R_{q}(x)=q x \bar{q}$. Consider the binary dihedral group $\widetilde{D}_{n}=u^{-1}\left(D_{n}\right)$. It fits in the following short exact sequence:

$$
1 \longrightarrow \mathbb{Z} / 2 \mathbb{Z} \longrightarrow \tilde{D}_{n} \longrightarrow D_{n} \longrightarrow 1,
$$


from which we get the 5-term exact sequence (see e.g. [5] p. 47, Exercise 6):

$$
H_{2}\left(\widetilde{D}_{n}\right) \longrightarrow H_{2}\left(D_{n}\right) \longrightarrow\left(H_{1}(\mathbb{Z} / 2 \mathbb{Z})\right)_{D_{n}} \longrightarrow H_{1}\left(\widetilde{D}_{n}\right) \longrightarrow H_{1}\left(D_{n}\right) \longrightarrow 0,
$$

where all the coefficients are in $\mathbb{Z}$ and $\left(H_{1}(\mathbb{Z} / 2 \mathbb{Z})\right)_{D_{n}}$ is the group of co-invariants under the $D_{n}$-action on $\mathbb{Z} / 2 \mathbb{Z}$ induced by conjugation by $\widetilde{D}_{n}$, hence we deduce $\left(H_{1}(\mathbb{Z} / 2 \mathbb{Z})\right)_{D_{n}}=H_{1}(\mathbb{Z} / 2 \mathbb{Z})$ since $\mathbb{Z} / 2 \mathbb{Z}$ is in the center of $\widetilde{D}_{n}$.

We have that $H_{2}\left(\widetilde{D}_{n}\right)=\{0\}$, since $\widetilde{D}_{n}$ is a finite subgroup of $\mathrm{SU}(2) \cong S^{3}$ (see [5] p. 47 , Exercise 7). Next, recall that, for any group $G, H_{1}(G, \mathbb{Z})$ is isomorphic to the abelianization $G^{\mathrm{ab}}$ (see [5] p. 36), hence (7) reduces to

$$
0 \longrightarrow H_{2}\left(D_{n}\right) \longrightarrow \mathbb{Z} / 2 \mathbb{Z} \longrightarrow \widetilde{D}_{n}^{\mathrm{ab}} \longrightarrow D_{n}^{\mathrm{ab}} \longrightarrow 0 .
$$

To conclude we show that $\operatorname{ker}\left(\widetilde{D}_{n}^{\mathrm{ab}} \rightarrow D_{n}^{\mathrm{ab}}\right)=\{0\}$ if and only if $n$ is even. With the imaginary units $\underline{i}, \underline{j}, \underline{k} \in \mathbb{H}$ let

$$
\xi=\cos \left(\frac{\pi}{n}\right)+\underline{k} \cdot \sin \left(\frac{\pi}{n}\right) \in u^{-1}(x) \quad \text { and } \quad \eta=\underline{j} \in u^{-1}(y) .
$$

Since $\left[\xi^{\ell}, \eta\right]=\xi^{2 \ell}$, for all $\ell$, we see that, if $n$ is odd, $\xi^{n} \notin\left[\widetilde{D}_{n}, \widetilde{D}_{n}\right]$, but $u\left(\xi^{n}\right)=1$ and hence $\operatorname{ker}\left(\widetilde{D}_{n}^{\mathrm{ab}} \rightarrow D_{n}^{\mathrm{ab}}\right) \neq\{0\}$. When $n$ is even, $\widetilde{D}_{n}^{\mathrm{ab}} \cong \mathbb{Z} / 2 \mathbb{Z} \times \mathbb{Z} / 2 \mathbb{Z}$ and the map $\widetilde{D}_{n}^{\mathrm{ab}} \rightarrow D_{n}^{\mathrm{ab}}$ is an isomorphism.

Using Lemma 3.2 from [40], we deduce the following

Corollary 4.2. Let $n \in \mathbb{N}, n \geq 4$ even. Then, the binary dihedral group $\widetilde{D}_{n}$ is a Schur cover of $D_{n}$ and the exact sequence (6) identifies $\mathbb{Z} / 2 \mathbb{Z}$ with $H_{2}\left(D_{n}, \mathbb{Z}\right)$. In particular, for any $\left(a_{1}, b_{1}, \ldots, a_{g^{\prime}}, b_{g^{\prime}}\right) \in\left(D_{n}\right)^{2 g^{\prime}}$ with $\prod_{1}^{g^{\prime}}\left[a_{i}, b_{i}\right]=1$, the image of $\prod_{1}^{g^{\prime}}\left[\widehat{a_{i}}, \widehat{b_{i}}\right] \in R \cap[F, F]$ in $H_{2}\left(D_{n}, \mathbb{Z}\right)=\frac{R \cap[F, F]}{[R, F]}$ is given by $\prod_{1}^{g^{\prime}}\left[\tilde{a}_{i}, \tilde{b}_{i}\right]$, where $\tilde{a}_{i}, \tilde{b}_{i} \in \widetilde{D}_{n}$ are liftings of $a_{i}, b_{i}$.

Next the union $\Gamma$ of non-trivial conjugacy classes comes into play. Recall that the set of reflections $\left\{x^{i} y \mid 0 \leq i<n\right\}$ is a single conjugacy class in case $n$ is odd, and splits into two conjugacy classes in case $n$ even according to the parity of $i$.

The conjugacy classes in the set of rotations $\left\{x^{i} \mid 0 \leq i<n\right\}$ are of the kind $\left\{x^{i}, x^{n-i}\right\}$ and contain two elements except for $i=0$ and $i=\frac{n}{2}$ in case $n$ is even.

Corollary 4.3. Let $\Gamma \subset D_{n}$ be a union of non-trivial conjugacy classes, $\Gamma \neq \emptyset$. Then $H_{2, \Gamma}\left(D_{n}\right)=\{0\}$ in the following cases: $n$ is odd; $n$ is even and $\Gamma$ contains some reflection; $n$ is even and $\Gamma$ contains the non-trivial central element. In the remaining case, $H_{2, \Gamma}\left(D_{n}\right)=\mathbb{Z} / 2 \mathbb{Z}$. 
Proof. If $n$ is odd, then $H_{2}\left(D_{n}, \mathbb{Z}\right)=\{0\}$ and hence $H_{2, \Gamma}\left(D_{n}\right)=\{0\}$ for any $\Gamma$ (Lemma 3.12).

If $n=2 k$ and $\Gamma$ contains some reflection, say $y \in \Gamma$, then $\left.\hat{y} \widehat{x^{k}} \hat{y}^{-1} \widehat{\left(x^{k}\right.}\right)^{-1}$ is in $R_{\Gamma} \cap[F, F]$. But the image of this element in $H_{2}\left(D_{n}, \mathbb{Z}\right)$ is not trivial (Corollary 4.2), hence $H_{2, \Gamma}\left(D_{n}\right)=\{0\}$ (Lemma 3.12). The same argument works if $x y \in \Gamma$.

Assume now $n=2 k$ and $x^{k} \in \Gamma$. Then $\widehat{x^{k}} \hat{y}\left(x^{k}\right)^{-1} \hat{y}^{-1} \in R_{\Gamma} \cap[F, F]$ and its image in $H_{2}\left(D_{n}, \mathbb{Z}\right)$ is not trivial, hence $H_{2, \Gamma}\left(D_{n}\right)=\{0\}$ also in this case.

Finally, if $n=2 k$ and $\Gamma \subset \mathbb{Z} / n \mathbb{Z} \backslash\left\{x^{k}\right\}$, then

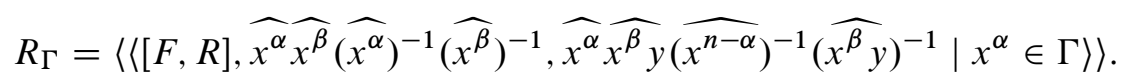

First we note that the image of $\widehat{x^{\alpha}} \widehat{x^{\beta}}\left(\widehat{x^{\alpha}}\right)^{-1}\left(\widehat{x^{\beta}}\right)^{-1}$ in $H_{2}\left(D_{n}, \mathbb{Z}\right)$ is 0 . Second, the elements $\widehat{x^{\alpha}} \widehat{x^{\beta}} y\left(\widehat{x^{n-\alpha}}\right)^{-1}\left(\widehat{x^{\beta} y}\right)^{-1}$ generate an abelian group modulo $[F, R]$. Last, the intersection of this subgroup with $[F, F] /[F, R]$ is generated by elements represented by

$$
\widehat{x^{\alpha}} \widehat{x^{\beta}} y\left(\widehat{x^{n-\alpha}}\right)^{-1} \widehat{x^{\beta} y^{-1}} \cdot \widehat{x^{n-\alpha}} \widehat{x^{\gamma} y} \widehat{x^{\alpha}-1}\left(\widehat{x^{\gamma} y}\right)^{-1} \text {. }
$$

It remains to show that these are trivial modulo $[F, R]$, in fact

$$
\begin{aligned}
& \left(\widehat{x^{\beta} y}\right)^{-1} \widehat{x^{\alpha}} \widehat{x^{\beta}} y\left(\widehat{x^{n-\alpha}}\right)^{-1} \cdot \widehat{x^{n-\alpha}} \widehat{x^{\gamma} y}\left(\widehat{x^{\alpha}}\right)^{-1}\left(\widehat{x^{\gamma} y}\right)^{-1} \\
& \equiv\left(\widehat{x^{\beta} y}\right)^{-1} \widehat{x^{\alpha} x^{\beta} y} \widehat{x^{\gamma} y}\left(\widehat{x^{\alpha}}\right)^{-1}\left(\widehat{x^{\gamma} y}\right)^{-1}
\end{aligned}
$$

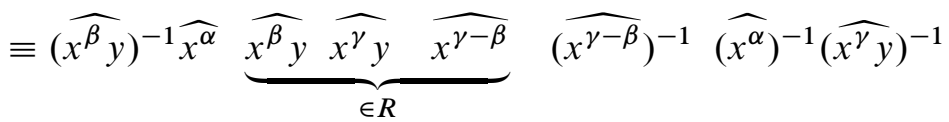

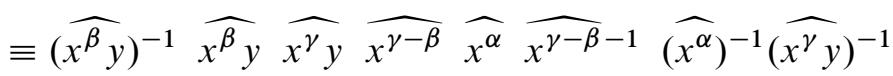

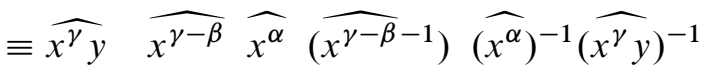

$$
\begin{aligned}
& \equiv \widehat{x^{\gamma-\beta}} \widehat{x^{\alpha}}\left(\widehat{x^{\gamma-\beta}}\right)^{-1} \widehat{x^{\alpha}-1} \text {. }
\end{aligned}
$$

This last element is trivial modulo $[F, R]$ as noted first. We deduce $\frac{R_{\Gamma} \cap[F, F]}{[F, R]}=\{0\}$ and hence $H_{2, \Gamma}\left(D_{n}\right) \cong H_{2}\left(D_{n}, \mathbb{Z}\right) \cong \mathbb{Z} / 2 \mathbb{Z}$, by Lemma 3.12. 


\section{The injectivity of $\hat{\varepsilon}$ when $G=D_{n}$}

Recall the following

Notation. For any Hurwitz vector $v=\left(c_{1}, \ldots, c_{d} ; a_{1}, b_{1}, \ldots, a_{g^{\prime}}, b_{g^{\prime}}\right) \in G^{d+2 g^{\prime}}$,

$$
\operatorname{ev}(v)=\prod_{i=1}^{d} c_{i} \cdot \prod_{j=1}^{g^{\prime}}\left[a_{j}, b_{j}\right] \in G
$$

while, if $c_{i} \neq 1$, for all $i, \varepsilon(v)=\operatorname{ev}(\hat{v}) \in G_{\Gamma_{v}}$, where $\hat{v} \in\left(G_{\Gamma_{v}}\right)^{d+2 g^{\prime}}$ is the tautological lifting (Definition 3.4).

In this section we prove the following

Theorem 5.1. Let $G=D_{n}$, the dihedral group of order $2 n$. For all $g^{\prime}, d$, the following results hold.

(i) The map

$$
\hat{\varepsilon}:\left(\operatorname{HS}\left(G ; g^{\prime}, d\right) / \operatorname{Aut}(G)\right) / \operatorname{Map}\left(g^{\prime}, d\right) \longrightarrow\left(G^{\cup}\right) / \operatorname{Aut}(G):=\left(\coprod_{\Gamma} G_{\Gamma}\right) / \operatorname{Aut}(G)
$$

is injective.

(ii) The image $\operatorname{Im}(\hat{\varepsilon})$ is the inverse image of $\operatorname{Im}(\hat{v})$ in $\left(\coprod_{\Gamma} K_{\Gamma}\right) / \operatorname{Aut}(G)$, where $K_{\Gamma}$ is defined in Definition 3.1. In other words, for any $\Gamma \subset G$, union of non trivial conjugacy classes, $\operatorname{Im}(\varepsilon) \cap G_{\Gamma}=H_{2, \Gamma}(G) \cdot\left(\operatorname{Im}(\varepsilon) \cap G_{\Gamma}\right)$.

(iii) For $g^{\prime} \geq 2, \operatorname{Im}(\hat{v})$ is just the set of admissible class functions $v$. For $g^{\prime}=1, \operatorname{Im}(\hat{v})$ is the union of the set of admissible class functions $v$ for which $\Gamma$ contains some reflection, together with a subset $\mathcal{S}$ of those for which $\Gamma$ generates a subgroup $H$ of index at most 2 in the subgroup $\mathcal{R}$ of rotations, $\mathcal{S}$ contains the subset for which $H=\mathcal{R}$.

To prove $(\mathrm{i})$, let $\left[v_{1}\right]_{\approx},\left[v_{2}\right]_{\approx} \in(\mathrm{HS}) / \approx$ be equivalence classes up to the combined action of $\operatorname{Aut}(G)$ and the mapping class group such that $\hat{\varepsilon}\left(\left[v_{1}\right]_{\approx}\right)=\hat{\varepsilon}\left(\left[v_{2}\right]_{\approx}\right)$. Then there exists an automorphism $f \in \operatorname{Aut}(G)$ such that $f\left(\Gamma_{v_{1}}\right)=\Gamma_{v_{2}}$ and $f\left(\varepsilon\left(v_{1}\right)\right)=\varepsilon\left(v_{2}\right)$. Hence, by Lemma 3.5, we assume without loss of generality $\Gamma_{v_{1}}=\Gamma_{v_{2}}=\Gamma$ and $\varepsilon\left(v_{1}\right)=\varepsilon\left(v_{2}\right)$, in particular

$$
\varepsilon\left(v_{1}\right) \cdot \varepsilon\left(v_{2}\right)^{-1}=0 \in H_{2, \Gamma}(G) .
$$


The outline of the proof is now the following. We address the following mutually exclusive cases: $\Gamma=\emptyset$ (the étale case); $\Gamma \neq \emptyset$ and contains some reflection; $\Gamma \neq \varnothing$ and does not contain reflections. In the first case, for each element of HS, we determine a normal form with respect to $\approx$, then we show that two different normal forms are distinguished by $H_{2}\left(D_{n}, \mathbb{Z}\right)$ (recall that $\operatorname{Aut}\left(D_{n}\right)$ acts trivially on $H_{2}\left(D_{n}, \mathbb{Z}\right)$ ). In the second case we will show that all Hurwitz generating systems with the same numerical invariants $\left(n, g^{\prime}\right.$ and $\nu$-type) are equivalent with respect to $\approx$ (this agrees with the fact $H_{2, \Gamma}\left(D_{n}\right)=\{0\}$ in this case). In the last case, for every $v \in$ HS, we determine a normal form $v^{\prime}$ with respect to $\approx$. We see that two different normal forms $v_{1}^{\prime}$ and $v_{2}^{\prime}$ have different invariants, $\varepsilon\left(v_{1}^{\prime}\right) \neq \varepsilon\left(v_{2}^{\prime}\right) \in G_{\Gamma}$. Finally we prove that $v_{1} \approx v_{2}$ if and only if there exists $f \in \operatorname{Aut}\left(D_{n}\right)$ such that $f(\Gamma)=\Gamma$ and $f\left(\varepsilon\left(v_{1}^{\prime}\right)\right)=\varepsilon\left(v_{2}^{\prime}\right)$. From this (i) follows. We refer to [10] for a useful description of $\operatorname{Aut}\left(D_{n}\right)$.

To prove claim (ii), we observe that for any $v \in \operatorname{HS}\left(D_{n} ; g^{\prime}, d\right)$ the orbit $\epsilon(v) \cdot H_{2, \Gamma_{v}}\left(D_{n}\right)$ is either $\{\varepsilon(v)\}$ or $\{\varepsilon(v),-\varepsilon(v)\}$ (cf. Corollary 4.3). In the proof of (i) we list all possible normal forms for Hurwitz generating systems and we will see that, in the case where $H_{2, \Gamma_{v}}\left(D_{n}\right) \cong \mathbb{Z} / 2 \mathbb{Z}=\{ \pm 1\}$, there exists $v^{\prime} \in \operatorname{HS}\left(D_{n} ; g^{\prime}, d\right)$ with $\Gamma_{v^{\prime}}=\Gamma_{v}$ and $\varepsilon\left(v^{\prime}\right) \cdot \varepsilon(v)^{-1}=-1$.

To prove (iii), observe that, given an admissible class function $v$, and elements $c_{1}, \ldots, c_{d}$ which yield the given function $v$, the product $c_{1} \cdots c_{d}:=c$ is in the commutator subgroup. However, in the dihedral group the commutator subgroup is equal to the set of commutators. Hence we may find $a_{1}, b_{1}$ such that $c^{-1}=\left[a_{1}, b_{1}\right]$, it suffices, if $c=x^{2 \delta}$, to take $a_{1}=x^{m} y, b_{1}=x^{m+\delta} y$.

If some $c_{j}$ is a reflection, without loss of generality, we may assume that $c_{j}=y$, hence, choosing $m=1$, we obtain that

$$
v:=\left(c_{1}, \ldots c_{d} ; x y, x^{1+\delta} y, 1, \ldots, 1\right)
$$

is a Hurwitz generating system. If $g^{\prime} \geq 2$, we can just take the Hurwitz generating system

$$
v:=\left(c_{1}, \ldots c_{d} ; y, x^{\delta} y, x, 1, \ldots, 1\right) .
$$

In the case where $g^{\prime}=1$ and all the $c_{j}$ are rotations, observe that

$$
v:=\left(c_{1}, \ldots c_{d} ; x^{m} y, x^{m+\delta} y\right)
$$

is a Hurwitz generating system if the dihedral group is generated by $H$ (the subgroup generated by the $c_{j}$ 's), together with $x^{m} y$ and $x^{\delta}$. This amounts to the condition that $H$ and $x^{\delta}$ generate the subgroup $\mathcal{R}$ of rotations. Since $c=x^{2 \delta} \in H$, we see that a necessary condition is that $H$ has index at most two in the group $\mathcal{R}$ of rotations, and a sufficient one is that $H=\mathcal{R}$. In the case where the index is exactly 
two, so that $n=2 h$ is even, the condition is that $\delta$ is an odd number. Observe that in this case if we replace some $c_{j}=x^{2 i}$ by its inverse, the number $\delta$ is replaced by $\delta-2 i$, so that condition is indeed a condition on the class function $v$.

Remark 5.2. For the case $g^{\prime}=0$ we defer to our previous article [10], where all the normal forms were given.

But we can give a direct description as follows.

For $g^{\prime}=0, \operatorname{Im}(\hat{v})$ is just the set of admissible class functions $v$ satisfying one of the following conditions:

(R) there are only two reflection classes, and the subgroup generated by the rotation classes is the whole $\mathcal{R}$;

(O) $n$ is odd and the class function $v$ takes value at least 4 on the reflection class;

(E) $n$ is even, there are at least four reflection classes and either the class function $v$ takes positive value on both reflection classes, or there exists a rotation class with odd exponent.

In fact, since we want a Hurwitz generating system, it is a necessary condition that there must be at least one reflection (and indeed an even number of reflections by admissibility). Take now an admissible class satisfying this restriction, and take $c_{1}, \ldots, c_{d}$ realizing the admissible class. Then their product $c$ is in the commutator subgroup, so it is any rotation in case $n$ is odd and a rotation of type $x^{2 i}$ in case $n$ is even.

Since there is a reflection, we replace the last reflection, say $c_{i}$, by $c_{i} c^{-1}$, and obtain thus an admissible Hurwitz system without changing the class function.

We must however have an admissible Hurwitz generating system, and we recall that there is an even number of reflections among the $c_{i}$ 's.

Up to automorphisms of $D_{n}$, we have reflections $y$ and $x^{m} y$ in the components of the Hurwitz vector.

If we have only two reflections $y, x^{m} y$, we are done if and only if the subgroup generated by the rotations is the whole subgroup $\mathcal{R}$, since $x^{-m} \in \mathcal{R}$.

Assume that $n$ is odd and there are 4 reflections: assume that the first four are $y, x^{m_{1}} y, x^{m_{2}} y, x^{m_{3}} y$ : then we can replace these (without changing the class function) by the following reflections $y, x y, x^{m_{2}-m_{1}+1} y, x^{m_{3}} y$ and we have obtained a Hurwitz generating system. 
Assume that $n$ is even and there are at least four reflections:

$$
y, \quad x^{m_{1}} y, \quad x^{m_{2}} y, \quad x^{m_{3}} y, \quad \ldots
$$

We can change the rotations by adding to $m_{i}$ an even integer $2 h_{i}$, in such a way that the sum $-h_{1}+h_{2}-h_{3} \ldots \equiv 0$. Hence if some $m_{i}$ is odd, we obtain $y$ and $x y$, and we have a generating system. If instead all $m_{i}$ 's are even, we obtain $y$ and $x^{2} y$, hence we are finally done if and only if there is a rotation with odd exponent.

Case 1: $\Gamma=\emptyset$ (the étale case). Here $H_{2, \Gamma}\left(D_{n}\right)=H_{2}\left(D_{n}, \mathbb{Z}\right)$, so $v \in \operatorname{HS}\left(D_{n}\right)$ implies $\varepsilon(v) \in H_{2}\left(D_{n}, \mathbb{Z}\right)$. In the following, we identify $H_{2}\left(D_{n}, \mathbb{Z}\right) \cong \mathbb{Z} / 2 \mathbb{Z}$ with $\{0,1\}$, when $n$ is even. Then we have the following result.

Proposition 5.3. Let $n, g^{\prime} \in \mathbb{N}$ with $n \geq 3, g^{\prime}>0$. Then, for any $v \in \operatorname{HS}\left(D_{n} ; g^{\prime}\right)$,

(i) $v \approx(y, 1, x, 1, \ldots, 1)$, if $n$ is odd or if $n$ is even and $\varepsilon(v)=0$;

(ii) $v \approx\left(y, x^{n / 2}, x, 1, \ldots, 1\right)$, if $n$ is even and $\varepsilon(v)=1$.

Proof. Let

$$
\bar{v}=v(\bmod \mathbb{Z} / n \mathbb{Z}) \in(\mathbb{Z} / 2 \mathbb{Z})^{2 g^{\prime}} .
$$

Notice that $\bar{v} \in \mathrm{HS}\left(\mathbb{Z} / 2 \mathbb{Z} ; g^{\prime}\right)$. Since the parameter space for étale $\mathbb{Z} / 2 \mathbb{Z}$-coverings of curves of a fixed genus is irreducible (see e.g. [16] Lemma 5.16, or [3], or [14], or [9], Theorem 2.4), there exists $\varphi \in \operatorname{Map}_{g^{\prime}}$ such that $\varphi \cdot \bar{v}=(1,0, \ldots, 0)$. Hence

$$
\varphi \cdot v=\left(x^{\ell_{1}} y, x^{m_{1}}, \ldots, x^{\ell_{g^{\prime}}}, x^{m_{g^{\prime}}}\right) .
$$

The condition $\operatorname{ev}(\varphi \cdot v)=1$ implies that $2 m_{1}=0(\bmod n)$. Hence $m_{1}=0$ or $m_{1}=\frac{n}{2}(\bmod n)$.

In the first case, which is the only possible if $n$ is odd,

$$
\varphi \cdot v=\left(x^{\ell_{1}} y, 1, x^{\ell_{2}}, \ldots, x^{\ell_{g^{\prime}}}, x^{m_{g^{\prime}}}\right) .
$$

Consider now $v^{\prime}:=\left(x^{\ell_{2}}, x^{m_{2}}, \ldots, x^{\ell_{g^{\prime}}}, x^{m_{g^{\prime}}}\right)$. As $v^{\prime} \in \mathrm{HS}\left(\mathbb{Z} / n \mathbb{Z} ; g^{\prime}-1\right)$, from the irreducibility of the parameter space of étale $\mathbb{Z} / n \mathbb{Z}$-coverings of curves of a fixed genus we deduce that there exists $\varphi^{\prime} \in \operatorname{Map}_{g^{\prime}-1}$ such that $\varphi^{\prime} \cdot v^{\prime}=\left(x^{\lambda}, 1, \ldots, 1\right)$, with $(\lambda, n)=1$ (see e.g. [16] Lemma 5.16, or [3], or [14], or [9], Theorem 2.4). Now, from Proposition A.3 it follows that there exists $\psi \in$ Map $_{g^{\prime}}$ such that

$$
\psi \cdot v=\left(x^{\ell_{1}} y, 1, x^{\lambda}, 1, \ldots, 1\right)
$$


We obtain the normal form (i) after operating with $\operatorname{Aut}\left(D_{n}\right)$. The fact that $\varepsilon(v)=0$ follows from a standard computation (cf. Corollary 4.2).

If $m_{1}=\frac{n}{2}(\bmod n)$, we have two subcases: $\left\langle x^{\ell_{2}}, x^{m_{2}}, \ldots, x^{\ell_{g^{\prime}}}, x^{m_{g^{\prime}}}\right\rangle=\mathbb{Z} / n \mathbb{Z}$ (which is the case when $n / 2$ is even), or $\left\langle x^{\ell_{2}}, x^{m_{2}}, \ldots, x^{\ell} g^{\prime}, x^{m_{g^{\prime}}}\right\rangle=\left\langle x^{2}\right\rangle$. Proceeding as in the case $m_{1}=0$ we reach the normal form (ii) in the first subcase, otherwise we obtain $\left(x^{\ell_{1}} y, x^{\frac{n}{2}}, x^{2}, 1, \ldots, 1\right)$. In the latter case, consider the transformation

$$
\left(a_{1}, b_{1}, a_{2}, b_{2}\right) \longmapsto\left(a_{2} a_{1}, b_{1}, b_{1} a_{2} b_{1}^{-1}, a_{2} b_{2} a_{2} b_{1}^{-1}\right),
$$

which is realized by $\mathrm{Map}_{2}$ as it preserves the relation $\prod_{1}^{2}\left[\alpha_{i}, \beta_{i}\right]=1$. Then extend it to $\Pi_{g^{\prime}}$ using Proposition A.3 and apply the transformation so obtained to $\left(x^{\ell_{1}} y, x^{\frac{n}{2}}, x^{2}, 1, \ldots, 1\right)$. We obtain:

$$
v \approx\left(x^{\ell_{1}+2} y, x^{\frac{n}{2}}, x^{2}, x^{4+\frac{n}{2}}, 1, \ldots, 1\right) .
$$

Since $\mathbb{Z} / n \mathbb{Z}=\left\langle x^{\frac{n}{2}}, x^{2}\right\rangle$, there exists $\psi \in \operatorname{Map}_{g^{\prime}}$ such that $\psi$ applied to the right hand side is $\left(x^{\ell_{1}+2} y, x^{\frac{n}{2}}, x, 1, \ldots, 1\right)$, therefore we obtain the normal form (ii). In both of these subcases we have $\varepsilon(v)=1$ (cf. Corollary 4.2).

Case 2: $\Gamma \neq \varnothing$ and contains some reflection. Let $v$ be a Hurwitz generating system, $v=\left(c_{1}, \ldots, c_{d} ; a_{1}, b_{1}, \ldots, a_{g^{\prime}}, b_{g^{\prime}}\right)$, such that $\left\{c_{1}, \ldots, c_{d}\right\}$ contains some reflection, actually an even number because any product of commutators in $D_{n}$ is a rotation. If $n$ is odd, all the reflections belong to the same conjugacy class, while when $n=2 k$ they are divided into two classes. Denote by $v_{y}$ (resp. $v_{x y}$ ) the number of $c_{i}$ 's in the class of $y$ (resp. $x y$ ). As the pair $\left(v_{y}, v_{x y}\right)$ is not $\operatorname{Aut}\left(D_{n}\right)$ invariant, we define $v_{1}, v_{2}$ by the property that $\left\{v_{1}, v_{2}\right\}=\left\{v_{y}, v_{x y}\right\}, v_{1} \leq v_{2}$ (in [10] we used the notation $h$ for $v_{1}, k$ for $v_{2}$ ). Recall that, under the above hypotheses, $H_{2, \Gamma}\left(D_{n}\right)=\{0\}$ (Corollary 4.3). Indeed we prove that all the $v$ 's with fixed $g^{\prime}, d, n$ and $\nu$-type are equivalent each other.

Proposition 5.4. Let $n, g^{\prime}, d \in \mathbb{N}$ with $n \geq 3, g^{\prime}, d>0$. Then, for any $v \in \operatorname{HS}\left(D_{n} ; g^{\prime}, d\right)$ such that $\Gamma_{v}$ contains some reflection,

(i) $v \approx\left(x^{\underline{r}}, x^{1-|\underline{\mid r}|} y, x y, y, \ldots, y ; x, 1, \ldots, 1,1\right)$, if $n$ is odd;

(ii) $v \approx(x^{\underline{r}}, \underbrace{x^{\varepsilon-|\underline{r}|} y, x y, \ldots, x y}_{\nu_{2}}, \underbrace{y, \ldots, y}_{\nu_{1}} ; x, 1, \ldots, 1,1)$, if $n$ is even.

Here $\underline{r}=\left(r_{1}, \ldots, r_{R}\right)$, where $R+v_{y}=d$ in case (i), $R+v_{y}+v_{x y}=d$ in case (ii), $0<r_{i} \leq r_{i+1} \leq \frac{n}{2}, x^{\underline{r}}=\left(x^{r_{1}}, \ldots, x^{r_{R}}\right),|\underline{r}|=\sum r_{i} \bmod n,\left\{v_{1}, v_{2}\right\}=$ $\left\{v_{y}, v_{x y}\right\}, v_{1} \leq v_{2}, \varepsilon \in\{0,1\}, \varepsilon+v_{2} \equiv 1 \bmod 2$. 
The idea of the proof is the following. Using the action of the unpermuted mapping class group $\operatorname{Map}^{u}\left(g^{\prime}, d+1\right)$ and the fact that at least one $c_{i}$ is a reflection, we prove that $v \sim\left(\tilde{c}_{1}, \ldots, \tilde{c}_{d} ; \tilde{a}_{1}, \tilde{b}_{1}, \ldots, \tilde{a}_{g^{\prime}}, \tilde{b}_{g^{\prime}}\right)$, with $\tilde{a}_{i}, \tilde{b}_{i} \in \mathbb{Z} / n \mathbb{Z}$, for any $i$. We collect in Appendix A the relevant facts that will be used about the action of $\operatorname{Map}^{u}\left(g^{\prime}, d+1\right)$ on the fundamental group. Then, using results about étale $\mathbb{Z} / n \mathbb{Z}$-covers, we deduce that $v \approx v^{\prime}:=\left(c_{1}^{\prime}, \ldots, c_{d}^{\prime} ; x, 1, \ldots, 1\right)$ (Lemma 5.5). At this point we can apply the main theorem of [10] to deduce that, acting with the braid group, it is possible to transform $v^{\prime}$ to the corresponding normal form. However, we will see that using the entry $x$ in $v^{\prime}$, the results in Appendix A and Lemma 2.1 of [10], we can transform directly $v^{\prime}$ in one of the above forms without using the normal forms for the $g^{\prime}=0$ case.

Lemma 5.5. Let $v$ be as in Proposition 5.4. Then

$$
v \approx v^{\prime}:=\left(c_{1}^{\prime}, \ldots, c_{d}^{\prime} ; x, 1, \ldots, 1,1\right) .
$$

Proof. Without loss of generality assume that $c_{d}$ is a reflection (otherwise act with the braid group). Then, if $a_{1}$ is a reflection, by Proposition A.2 (i), there exists $\varphi \in \operatorname{Map}^{u}\left(g^{\prime}, d+1\right)$ such that

$$
\varphi \cdot v=\left(c_{1}, \ldots, c_{d-1},\left(c_{d} a_{1} b_{1} a_{1}^{-1}\right) c_{d}\left(c_{d} a_{1} b_{1} a_{1}^{-1}\right)^{-1} ; c_{d} a_{1}, b_{1}, \ldots, a_{g^{\prime}}, b_{g^{\prime}}\right) .
$$

While, if $a_{1}$ is a rotation and $b_{1}$ is a reflection, by Proposition A.2 (ii) we have that there exists $\varphi \in \operatorname{Map}^{u}\left(g^{\prime}, d+1\right)$ such that

$$
\varphi \cdot v=\left(c_{1}, \ldots,\left(c_{d}\left[a_{1}, b_{1}\right] a_{1}^{-1}\right) c_{d}\left(c_{d}\left[a_{1}, b_{1}\right] a_{1}^{-1}\right)^{-1} ; a_{1},\left(a_{1}^{-1} c_{d} a_{1}\right) b_{1}, \ldots, b_{g^{\prime}}\right) .
$$

Notice that in both cases the $d$-th entry of $\varphi \cdot v$ is a reflection and that $c_{d} a_{1}$, $\left(a_{1}^{-1} c_{d} a_{1}\right) b_{1} \in \mathbb{Z} / n \mathbb{Z}$. Proceeding in this way we get $\psi \in \operatorname{Map}^{u}\left(g^{\prime}, d+1\right)$ such that $(\psi \cdot v)_{i} \in \mathbb{Z} / n \mathbb{Z}, i=d+1, \ldots, 2 g^{\prime}$.

Next, by the main theorem in [9], we conclude that

$$
\psi \cdot v \approx\left(\tilde{c}_{1}, \ldots, \tilde{c}_{d} ; x^{\alpha}, 1, \ldots, 1\right)
$$

We can further assume $(\alpha, n)=1$. Otherwise, since $D_{n}=\left\langle x^{\alpha}, \tilde{c}_{1}, \ldots, \tilde{c}_{d}\right\rangle$, there exists $x^{\beta} \in\left\langle\tilde{c}_{1}, \ldots, \tilde{c}_{d}\right\rangle$ such that $\mathbb{Z} / n \mathbb{Z}=\left\langle x^{\alpha+\beta}\right\rangle$. Using Proposition A.2 (i) and the braid group, we can multiply $x^{\alpha}$ by any element of $\left\langle\tilde{c}_{1}, \ldots, \tilde{c}_{d}\right\rangle$. The claim now follows by applying $\operatorname{Aut}\left(D_{n}\right)$. 
We now complete the proof of Proposition 5.4. Let $v^{\prime}$ be as in Lemma 5.5 and let $2 N$ be the number of reflections in $\left\{c_{1}^{\prime}, \ldots, c_{d}^{\prime}\right\}$. Applying Lemma 2.1 of [10] we have

$$
v^{\prime} \approx\left(x^{\underline{r}}, x^{\beta} y, x^{\alpha} y, x^{j_{N-1}} y, x^{j_{N-1}} y, \ldots, x^{j_{1}} y, x^{j_{1}} y ; x, 1, \ldots, 1\right),
$$

where $\underline{r}=\left(r_{1}, \ldots, r_{R}\right), 0<r_{i} \leq r_{i+1} \leq \frac{n}{2}, x^{\underline{r}}=\left(x^{r_{1}}, \ldots, x^{r_{R}}\right)$.

If $N=1$ the result is clear. Otherwise we conjugate by $x$ simultaneously the entries of each pair $\left(x^{j_{k}} y, x^{j_{k}} y\right)$ in (9) without changing the other components, hence we obtain

$$
\begin{gathered}
v^{\prime} \sim\left(x^{\underline{r}}, x^{\beta} y, x^{\alpha} y, x^{j_{N-1}+2 \ell_{N-1}} y, x^{j_{N-1}+2 \ell_{N-1}} y, \ldots,\right. \\
\left.x^{j_{1}+2 \ell_{1}} y, x^{j_{1}+2 \ell_{1}} y ; x, 1, \ldots, 1\right)
\end{gathered}
$$

for any $\ell_{1}, \ldots, \ell_{N-1} \in \mathbb{Z}$.

The equivalence (10) can be proven as follows. We have

$$
\begin{aligned}
v^{\prime} & \sim\left(x^{\underline{r}}, x^{\beta} y, \ldots, x^{j_{1}} y,\left(x^{j_{1}} y x^{-1}\right) x^{j_{1}} y\left(x^{j_{1}} y x^{-1}\right)^{-1} ; x, x^{-1} x^{j_{1}} y x, 1, \ldots, 1\right) \\
& \sim\left(x^{\underline{r}}, x^{\beta} y, \ldots,\left(x^{-1}\right) x^{j_{1}} y(x), x^{j_{1}} y ; x, x^{-1} x^{j_{1}} y x, 1, \ldots, 1\right) \\
& \sim\left(x^{\underline{r}}, x^{\beta} y, \ldots,\left(x^{-1}\right) x^{j_{1}} y(x),\left(x^{-1}\right) x^{j_{1}} y(x) ; x, 1, \ldots, 1\right),
\end{aligned}
$$

where the first and the third equivalences are given by $\xi$-twists as in Proposition A.2 (ii), while the second is a braid twist between the last two components. Iterating these steps we can conjugate by any power of $x$ the entries of $\left(x^{j_{1}} y, x^{j_{1}} y\right)$ simultaneously. By Lemma 2.3 in [10] we can move $\left(x^{j_{k}} y, x^{j_{k}} y\right)$ to the right and then conjugate its entries by any power of $x$ as before. This proves (10).

If $n$ is odd, choose $\ell_{i}$ in (10) such that $j_{i}+2 \ell_{i}=\alpha-1(\bmod n)$, then apply the automorphism $x^{\alpha-1} y \mapsto y, x \mapsto x$ to obtain (i).

Assume now that $n$ is even. Without loss of generality we have that $v_{1}=v_{y} \leq$ $v_{x y}=v_{2}$ (otherwise apply $\operatorname{Aut}\left(D_{n}\right)$ ). Assume further that $x^{\beta} y$ in (9) is conjugate to $x y$.

If $x^{\alpha} y$ is conjugate to $x y$, choose $\ell_{i}$ such that $j_{i}+2 \ell_{i}=\alpha$ or $j_{i}+2 \ell_{i}=\alpha-1$ $(\bmod n)$, so $(10)$ becomes:

$$
v^{\prime} \sim\left(x^{\underline{r}}, x^{\beta} y, x^{\alpha} y, x^{\alpha} y, \ldots, x^{\alpha} y, x^{\alpha-1} y, \ldots, x^{\alpha-1} y ; x, 1, \ldots, 1\right) .
$$

We obtain the normal form (ii) after applying the automorphism

$$
x^{\alpha} y \longmapsto x y, \quad x \mapsto x .
$$

The remaining case, where $x^{\alpha} y$ is conjugate to $y$, is similar.

Notice that (10) follows also from Lemma 2.1 in [27] (see also [23]), which applies to a more general situation. Since we don't need the whole strength of that result, we preferred to give a complete proof in our case. 
Case 3: $\Gamma \neq \emptyset$ and does not contain reflections. We prove the following

Proposition 5.6. Let $v, v^{\prime} \in \operatorname{HS}\left(D_{n} ; g^{\prime}, d\right)$ with $\Gamma_{v}, \Gamma_{v^{\prime}} \subset \mathbb{Z} / n \mathbb{Z}$. Then $v \approx v^{\prime}$ if and only if there exists $f \in \operatorname{Aut}\left(D_{n}\right)$ such that $f\left(\Gamma_{v}\right)=\Gamma_{v^{\prime}}$ and $f(\varepsilon(v))=\varepsilon\left(v^{\prime}\right)$.

The "only if" part is clear. So assume $\Gamma_{v}=\Gamma_{v^{\prime}}=: \Gamma$ and the existence of $f$ as in the statement. We prove that $v \approx v^{\prime}$. This is achieved after considering three cases: $n$ is odd; $n=2 k$ and $x^{k} \in \Gamma, n=2 k$ and $x^{k} \notin \Gamma$. In the first two cases we determine a normal form, with respect to $\approx$, for each such element of $\operatorname{HS}\left(D_{n} ; g^{\prime}, d\right)$, and then we show that two such elements are equivalent if and only if they have the same normal form. Notice that in both cases $H_{2, \Gamma}\left(D_{n}\right)=\{0\}$ (Corollary 4.3). In the last case, for any such $v \in \operatorname{HS}\left(D_{n} ; g^{\prime}, d\right)$, we determine a normal form $v^{\prime}$, with respect to the action of $\operatorname{Map}\left(g^{\prime}, d\right)$ and then we show that $v_{1} \approx v_{2}$ if and only if there exists $f \in \operatorname{Aut}\left(D_{n}\right)$ such that $f(\Gamma)=\Gamma$ and $f\left(\varepsilon\left(v_{1}^{\prime}\right)\right)=\varepsilon\left(v_{2}^{\prime}\right)$. Notice that, in this case $H_{2, \Gamma}\left(D_{n}\right) \cong \mathbb{Z} / 2 \mathbb{Z}$ (Corollary 4.3).

Notation. Let $v=\left(c_{1}, \ldots, c_{d} ; a_{1}, b_{1}, \ldots, a_{g^{\prime}}, b_{g^{\prime}}\right) \in D_{n}^{d+2 g^{\prime}}$ be a Hurwitz generating system such that $\Gamma_{v} \subset \mathbb{Z} / n \mathbb{Z}$, i.e. $c_{i} \in \mathbb{Z} / n \mathbb{Z}$, for all $i$. We denote by $H=\left\langle c_{1}, \ldots, c_{d}\right\rangle \subset D_{n}$ the subgroup generated by the $c_{i}$ 's. Note that, under the above hypotheses, $H$ is normal and it is contained in $\mathbb{Z} / n \mathbb{Z}$. Set $G^{\prime}:=D_{n} / H$. Then $G^{\prime}$ is a dihedral group $D_{m}, m \geq 3$, or is isomorphic to $\mathbb{Z} / 2 \mathbb{Z} \times \mathbb{Z} / 2 \mathbb{Z}$, or to $\mathbb{Z} / 2 \mathbb{Z}$.

Lemma 5.7. Let $n \in \mathbb{N}, n \geq 3$ odd. Let $v \in \operatorname{HS}\left(D_{n} ; g^{\prime}, d\right)$ with $\Gamma_{v} \subset \mathbb{Z} / n \mathbb{Z}$. Then

$$
v \approx\left(x^{\underline{r}} ; y, x^{h}, x, 1, \ldots, 1\right),
$$

where $\underline{r}=\left(r_{1}, \ldots, r_{d}\right), x^{\underline{r}}=\left(x^{r_{1}}, \ldots, x^{r_{d}}\right), r_{1} \leq \cdots \leq r_{d}<\frac{n}{2}$ and $2 h=\sum_{1}^{d} r_{i}, \bmod n$.

Proof. Let us consider

$$
\bar{v}:=\left(\overline{a_{1}}, \overline{b_{1}}, \ldots, \overline{a_{g^{\prime}}}, \overline{b_{g^{\prime}}}\right) \in \operatorname{HS}\left(G^{\prime} ; g^{\prime}, 0\right),
$$

where $\overline{a_{i}}=a_{i}(\bmod H), \overline{b_{i}}=b_{i}(\bmod H)$. By Proposition 5.3 and by the analogous results for cyclic and $(\mathbb{Z} / 2 \mathbb{Z} \times \mathbb{Z} / 2 \mathbb{Z})$-covers, there exists $\varphi \in \operatorname{Map}_{g^{\prime}}$ such that

$$
\varphi \cdot \bar{v}=(\bar{y}, 1, \bar{x}, 1, \ldots, 1) .
$$

By Proposition A.3, there exists $\tilde{\varphi} \in \operatorname{Map}\left(g^{\prime}, d\right)$ with

$$
\tilde{\varphi} \cdot v=\left(c_{1}, \ldots, c_{d} ; x^{\ell_{1}} y, x^{m_{1}}, x^{\ell_{2}}, \ldots, x^{m_{g^{\prime}}}\right),
$$

where $x^{m_{i}} \in H$, for all $i, x^{\ell_{2}}=x(\bmod H)$ and $x^{\ell_{i}} \in H$, for all $i>2$. 
We now apply the $\xi$-twists as in Proposition A.2 (i) with $\ell=2,3, \ldots, g^{\prime}$ and we deduce that we can multiply all the $x^{\ell_{i}}, i>1$, by any element of $H$. Hence there exists $\psi \in \operatorname{Map}^{u}\left(g^{\prime}, d+1\right)$ such that

$$
\psi \cdot \tilde{\varphi} \cdot v=\left(c_{1}, \ldots, c_{d} ; x^{\ell_{1}} y, x^{m_{1}}, x, x^{m_{2}}, 1, x^{m_{3}}, \ldots, 1, x^{m_{g^{\prime}}}\right) .
$$

Similarly, using Proposition A.2 (ii), we get:

$$
v \approx\left(c_{1}, \ldots, c_{d} ; x^{\ell_{1}} y, x^{m_{1}}, x, 1, \ldots, 1,1\right) .
$$

Now, for any $i=1, \ldots, d$, consider $c_{i}=x^{s_{i}}$. If $s_{i}<\frac{n}{2}$, set $r_{i}=s_{i}$, otherwise use the braid group to move $c_{i}$ to the $d$-th position and then apply Proposition A.2 (ii) with $\ell=1$. After this, $c_{i}$ becomes $c_{i}^{-1}=x^{n-s_{i}}$, then set $r_{i}=n-s_{i}$. Finally, using the braid group, we can order the $c_{i}$ 's such that $r_{i} \leq r_{i+1}$.

So, we have proved that

$$
v \sim\left(x^{\underline{r}} ; x^{\lambda_{1}} y, x^{\mu_{1}}, x, 1, \ldots, 1\right),
$$

with $r_{1} \leq \cdots \leq r_{d}<\frac{n}{2}$. Now the condition ev $(v)=1$ implies that $2 \mu_{1}=\sum_{1}^{d} r_{i}$ $(\bmod n)$, therefore set $h:=\mu_{1}(\bmod n)$.

We reach the normal form after applying the automorphism $x^{\lambda_{1}} y \mapsto y$, $x \mapsto x$.

Lemma 5.8. Let $n=2 k \in \mathbb{N}$ and let $v \in \operatorname{HS}\left(D_{n} ; g^{\prime}, d\right)$ with $x^{k} \in \Gamma_{v} \subset \mathbb{Z} / n \mathbb{Z}$. Then

$$
v \approx\left(x^{\underline{r}} ; y, x^{h}, x, 1, \ldots, 1\right),
$$

where $\underline{r}=\left(r_{1}, \ldots, r_{d}\right), x^{\underline{r}}=\left(x^{r_{1}}, \ldots, x^{r_{d}}\right), r_{1} \leq \cdots \leq r_{d}=k, 2 h=\sum_{1}^{d} r_{i}$ $(\bmod n)$ and $h<k$.

Proof. Proceeding as in the proof of Lemma 5.7, there exists $\varphi \in \operatorname{Map}\left(g^{\prime}, d\right)$ such that

$$
\varphi \cdot v=\left(c_{1}, \ldots, c_{d} ; x^{\ell_{1}} y, x^{m_{1}}, x^{\ell_{2}}, \ldots, x^{m_{g^{\prime}}}\right),
$$

where $x^{m_{i}} \in H$, for all $i>1, x^{\ell_{2}}=x(\bmod H)$ and $x^{\ell_{i}} \in H$, for all $i>2$.

Since we can multiply all the $x^{\ell_{i}}, i>1$, by any element of $H$ (apply Proposition A.2 (i) with $\left.\ell=2,3, \ldots, g^{\prime}\right)$, we have that there exists $\psi \in \operatorname{Map}^{u}\left(g^{\prime}, d+1\right)$ such that

$$
\psi \cdot \varphi \cdot v=\left(c_{1}, \ldots, c_{d} ; x^{\ell_{1}} y, x^{m_{1}}, x, x^{m_{2}}, 1, x^{m_{3}}, \ldots, 1, x^{m_{g^{\prime}}}\right) .
$$

Similarly, using Proposition A.2 (ii), we get

$$
v \approx\left(c_{1}, \ldots, c_{d} ; x^{\ell_{1}} y, x^{m_{1}}, x, 1, \ldots, 1,1\right) .
$$


Now, for any $i=1, \ldots, d$, consider $c_{i}=x^{s_{i}}$. If $s_{i} \leq k$, set $r_{i}=s_{i}$, otherwise use the braid group to move $c_{i}$ to the $d$-th position and then apply Proposition A.2 (ii) with $\ell=1$. In this way $c_{i}$ becomes $c_{i}^{-1}$ and so set $r_{i}=2 k-s_{i}$.

So, we have proved that

$$
v \approx\left(x^{\underline{r}} ; x^{\lambda_{1}} y, x^{\mu_{1}}, x, 1, \ldots, 1\right),
$$

with $r_{i} \leq k$, for all $i$. Now the condition $\operatorname{ev}(v)=1$ implies that $2 \mu_{1}=\sum_{1}^{d} r_{i}$ $(\bmod n)$ ). If $\mu_{1}<k$, set $h=\mu_{1}$. Otherwise, apply braid group transformations to achieve the ordering $r_{i} \leq r_{i+1}$, for all $i \leq d-1$. By hypotheses $r_{d}=k$ and we apply Proposition A.2 with $\ell=1$. Since $x^{k}$ is central, this operation does not change $r_{d}$, while $x^{\mu_{1}}$ becomes $x^{\mu_{1}+k}$. Set $h=\mu_{1}+k(\bmod n)$.

Finally apply the appropriate element of $\operatorname{Aut}\left(D_{n}\right)$ to reach the normal form.

We now consider the last case.

Lemma 5.9. Let $n=2 k \in \mathbb{N}$ and let $v \in \operatorname{HS}\left(D_{n} ; g^{\prime}, d\right)$ with $\Gamma \subset \mathbb{Z} / n \mathbb{Z} \backslash\left\{x^{k}\right\}$.

(i) There is an equivalence

$$
v \approx v^{\prime}:=\left(x^{\underline{r}} ; y, x^{h}, x, 1, \ldots, 1\right),
$$

where $\underline{r}=\left(r_{1}, \ldots, r_{d}\right), x^{\underline{r}}=\left(x^{r_{1}}, \ldots, x^{r_{d}}\right), r_{1} \leq \cdots \leq r_{d}<k$, and $2 h=\sum_{1}^{d} r_{i} \bmod n$.

(ii) Let $v_{1}^{\prime}=\left(x^{\underline{r}} ; y, x^{h}, x, 1, \ldots, 1\right)$ and $v_{2}^{\prime}=\left(x^{\underline{r}} ; y, x^{h+k}, x, 1, \ldots, 1\right)$, then $\varepsilon\left(v_{1}^{\prime}\right) \neq \varepsilon\left(v_{2}^{\prime}\right) \in\left(D_{n}\right)_{\Gamma}$.

(iii) $v_{1}^{\prime} \approx v_{2}^{\prime}$ if and only if there exists $f \in \operatorname{Aut}\left(D_{n}\right)$ such that $f(\Gamma)=\Gamma$ and $f\left(\varepsilon\left(v_{1}^{\prime}\right)\right)=\varepsilon\left(v_{2}^{\prime}\right)$.

Proof. The proof of (i) is the same as that of the previous lemma. Since in this case $x^{k} \notin \Gamma$, we can not achieve $h \leq k$.

To prove (ii) recall that $\varepsilon(v):=\operatorname{ev}(\hat{v}) \in\left(D_{n}\right)_{\Gamma}$. So, if $\operatorname{ev}\left(\widehat{v_{1}^{\prime}}\right)=\operatorname{ev}\left(\widehat{v_{2}^{\prime}}\right)$, then $\operatorname{ev}\left(\widehat{v_{2}^{\prime}}\right)^{-1} \cdot \operatorname{ev}\left(\widehat{v_{1}^{\prime}}\right)=0 \in H_{2, \Gamma}\left(D_{n}\right)$. But now a direct computation shows that $\operatorname{ev}\left(\widehat{v_{2}^{\prime}}\right)^{-1} \cdot \operatorname{ev}\left(\widehat{v_{1}^{\prime}}\right) \neq 0$ (Corollary 4.2), a contradiction.

(iii) The "only if" part is clear. So, assume that there exists $f \in \operatorname{Aut}\left(D_{n}\right)$ such that $f(\Gamma)=\Gamma$ and $f\left(\varepsilon\left(v_{1}^{\prime}\right)\right)=\varepsilon\left(v_{2}^{\prime}\right)$. Since $f\left(\varepsilon\left(v_{1}^{\prime}\right)\right)=\varepsilon\left(f\left(v_{1}^{\prime}\right)\right)$, we have $\varepsilon\left(f\left(v_{1}^{\prime}\right)\right)=\varepsilon\left(v_{2}^{\prime}\right)$ and so $v_{2}^{\prime}$ and $f\left(v_{1}^{\prime}\right)$ have the same $v$-type (Remark 3.10). From (i) and (ii) we deduce that

$$
f\left(v_{1}^{\prime}\right) \approx\left(x^{-} ; x^{\lambda_{1}} y, x^{h+k}, x, 1, \ldots, 1\right) .
$$


Hence, using the automorphism $x^{\lambda_{1}} y \mapsto y, x \mapsto x$, we have that $f\left(v_{1}^{\prime}\right) \approx v_{2}^{\prime}$ and so the claim follows.

\section{Appendixes}

\section{A. Automorphisms of surface-groups}

We collect in this appendix some facts about mapping class groups and their action on fundamental groups. They should be well known to experts, we include them here for completeness.

Let $Y$ be a compact Riemann surface of genus $g^{\prime}$ and let $\mathcal{B}=\left\{y_{1}, \ldots, y_{d}\right\} \subset Y$ be a finite subset of cardinality $d$. After the choice of a geometric basis of $Y \backslash \mathcal{B}$, we have the following presentation of the fundamental group:

$$
\pi_{1}\left(Y \backslash \mathcal{B}, y_{0}\right)=\left\langle\gamma_{1}, \ldots, \gamma_{d}, \alpha_{1}, \beta_{1}, \ldots, \alpha_{g^{\prime}}, \beta_{g^{\prime}} \mid \gamma_{1} \cdots \gamma_{d} \cdot \Pi_{i=1}^{g^{\prime}}\left[\alpha_{i}, \beta_{i}\right]=1\right\rangle .
$$

Following [4], there is a short exact sequence

$$
1 \longrightarrow \pi_{1}\left(Y \backslash \mathcal{B}, y_{0}\right) \stackrel{\Xi}{\longrightarrow} \operatorname{Map}^{u}\left(Y,\left\{y_{0}, y_{1}, \ldots, y_{d}\right\}\right) \longrightarrow \operatorname{Map}^{u}(Y, \mathcal{B}) \longrightarrow 1
$$

which induces an injective group homomorphism

$$
\operatorname{Map}^{u}(Y, \mathcal{B}) \longrightarrow \operatorname{Out}\left(\pi_{1}\left(Y \backslash \mathcal{B}, y_{0}\right)\right)
$$

([4], Theorem 4). The map $\Xi$ is defined as follows. Let $[c] \in \pi_{1}\left(Y \backslash \mathcal{B}, y_{0}\right)$ be an element of the geometric basis and let

$$
c:[0,2 \pi] \longrightarrow Y \backslash \mathcal{B}
$$

be a simple, smooth loop based at $y_{0}$, representing $[c]$. Let $\Xi([c])$ be the isotopy class of the $\xi$-twist, $\xi_{c}$. Then extend $\Xi$ to the whole group as an homomorphism. Recall that the $\xi$-twist, $\xi_{c}$, can be defined as follows. Let $N \subset Y \backslash \mathcal{B}$ be a tubular neighborhood of $c$ and let $e: A \rightarrow N$ be a diffeomorphism between the annulus $A=\left\{z=r e^{i \theta} \in \mathbb{C} \mid 1 \leq r \leq 2\right\}$ and $N$ such that $e\left(\frac{3}{2}, \theta\right)=c(\theta)$. Define

$$
h: A \longrightarrow A
$$

as follows:

$$
h(r, \theta)= \begin{cases}(r, \theta+4 \pi(r-1)), & 1 \leq r \leq \frac{3}{2} \\ (r, \theta+4 \pi(2-r)), & \frac{3}{2} \leq r \leq 2\end{cases}
$$




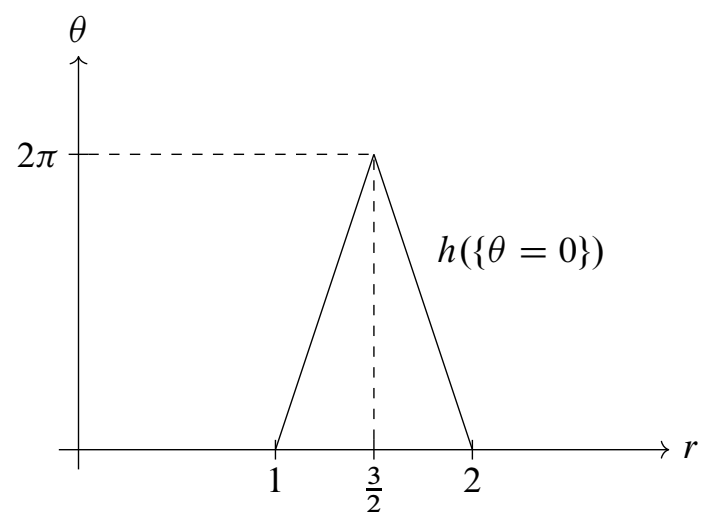

Then $h$ is a diffeomorphism which is the identity when $r=1, \frac{3}{2}, 2$. Finally, define $\xi_{c}: Y \rightarrow Y$ as the identity on $Y \backslash N$ and as $e \circ h \circ e^{-1}$ on $N$.

From the sequence (11), it follows that $\pi_{1}\left(Y \backslash \mathcal{B}, y_{0}\right)$ is isomorphic through $\Xi$ to a normal subgroup of $\operatorname{Map}^{u}\left(Y,\left\{y_{0}, y_{1}, \ldots, y_{d}\right\}\right)$, hence we get an action by conjugation of $\operatorname{Map}^{u}\left(Y,\left\{y_{0}, y_{1}, \ldots, y_{d}\right\}\right)$ on $\pi_{1}\left(Y \backslash \mathcal{B}, y_{0}\right)$ :

$$
[f] \cdot\left[\xi_{c}\right]=\left[f \circ \xi_{c} \circ f^{-1}\right] .
$$

We have:

Lemma A.1. For any $[f] \in \operatorname{Map}^{u}\left(Y,\left\{y_{0}, y_{1}, \ldots, y_{d}\right\}\right)$ and $[c] \in \pi_{1}\left(Y \backslash \mathcal{B}, y_{0}\right)$,

$$
[f] \cdot\left[\xi_{c}\right]=\left[\xi_{f_{\#}(c)}\right],
$$

where $f_{\#}(c)(\theta)=(f \circ c)(\theta)$.

Proof. Observe

$$
f \circ \xi_{c} \circ f^{-1}= \begin{cases}(f \circ e) \circ h \circ(f \circ e)^{-1} & \text { on } N, \\ \operatorname{Id} & \text { on } Y \backslash N .\end{cases}
$$

The result then follows because $f \circ e: A \rightarrow Y$ is a tubular neighborhood of $f_{\#}(c)$.

One can define, in the same way, $\xi$-twists with respect to loops that are not based at $y_{0}$ and Lemma A.1 is still valid. In the following result we give the action of $\xi$-twists around certain loops in terms of a given geometric basis of $\pi_{1}\left(Y \backslash \mathcal{B}, y_{0}\right)$. 
Proposition A.2. Let $\gamma_{1}, \ldots, \gamma_{d}, \alpha_{1}, \beta_{1}, \ldots, \alpha_{g^{\prime}}, \beta_{g^{\prime}}$ be a fixed geometric basis of $\pi_{1}\left(Y \backslash \mathcal{B}, y_{0}\right)$.

(i) Let $c \subset Y \backslash \mathcal{B}$ be the loop in Figure 1, image of the two sides of the angle inside the polygon with vertex $y_{d}$. Set

$$
u=\prod_{k=1}^{\ell-1}\left[\alpha_{k}, \beta_{k}\right] .
$$

Then

$$
\begin{aligned}
& \left(\xi_{c}\right)_{*}\left(\alpha_{\ell}\right)=u^{-1} \gamma_{d} u \alpha_{\ell} \\
& \left(\xi_{c}\right)_{*}\left(\gamma_{d}\right)=\left(\gamma_{d} u \alpha_{\ell} \beta_{\ell} \alpha_{\ell}^{-1} u^{-1}\right) \gamma_{d}\left(\gamma_{d} u \alpha_{\ell} \beta_{\ell} \alpha_{\ell}^{-1} u^{-1}\right)^{-1} \\
& \left(\xi_{c}\right)_{*}\left(\alpha_{i}\right)=\alpha_{i} \quad(i \neq \ell) \\
& \left(\xi_{c}\right)_{*}\left(\beta_{i}\right)=\beta_{i} \quad(\text { for all } i) \\
& \left(\xi_{c}\right)_{*}\left(\gamma_{j}\right)=\gamma_{j} \quad(j \neq d) .
\end{aligned}
$$

(ii) Let $c \subset Y \backslash \mathcal{B}$ be the loop in Figure 2, image of the two sides of the angle inside the polygon with vertex $y_{d}$. Set

$$
u=\prod_{k=1}^{\ell-1}\left[\alpha_{k}, \beta_{k}\right] .
$$

Then

$$
\begin{aligned}
& \left(\xi_{c}\right)_{*}\left(\beta_{\ell}\right)=\alpha_{\ell}^{-1} u^{-1} \gamma_{d} u \alpha_{\ell} \beta_{\ell} ; \\
& \left(\xi_{c}\right)_{*}\left(\gamma_{d}\right)=\left(\gamma_{d} u\left[\alpha_{\ell}, \beta_{\ell}\right] \alpha_{\ell}^{-1} u^{-1}\right) \gamma_{d}\left(\gamma_{d} u\left[\alpha_{\ell}, \beta_{\ell}\right] \alpha_{\ell}^{-1} u^{-1}\right)^{-1} \\
& \left(\xi_{c}\right)_{*}\left(\beta_{i}\right)=\beta_{i} \quad(i \neq \ell) \\
& \left(\xi_{c}\right)_{*}\left(\alpha_{i}\right)=\alpha_{i} \quad(\text { for all } i) ; \\
& \left(\xi_{c}\right)_{*}\left(\gamma_{j}\right)=\gamma_{j} \quad(j \neq d) .
\end{aligned}
$$

Proof. (i) The image of $\alpha_{\ell}$ under $\xi_{c}$ is drawn in Figure 3. From this it follows the formula for $\left(\xi_{c}\right)_{*}\left(\alpha_{\ell}\right)$. Since $\xi_{c}$ is the identity outside a small tubular neighborhood of $c$,

$$
\begin{array}{ll}
\left(\xi_{c}\right)_{*}\left(\alpha_{i}\right)=\alpha_{i} & (i \neq \ell), \\
\left(\xi_{c}\right)_{*}\left(\beta_{i}\right)=\beta_{i} & (\text { for all } i), \\
\left(\xi_{c}\right)_{*}\left(\gamma_{j}\right)=\gamma_{j} & (j \neq d) .
\end{array}
$$


The formula for $\left(\xi_{c}\right)_{*}\left(\gamma_{d}\right)$ is now a consequence of $\gamma_{1} \cdots \gamma_{d} \prod_{i=1}^{g^{\prime}}\left[\alpha_{i}, \beta_{i}\right]=1$, since the product $\gamma_{1} \cdots \cdots \gamma_{d} \prod_{i=1}^{g^{\prime}}\left[\alpha_{i}, \beta_{i}\right]$ must be left fixed.

The proof of (ii) is similar.

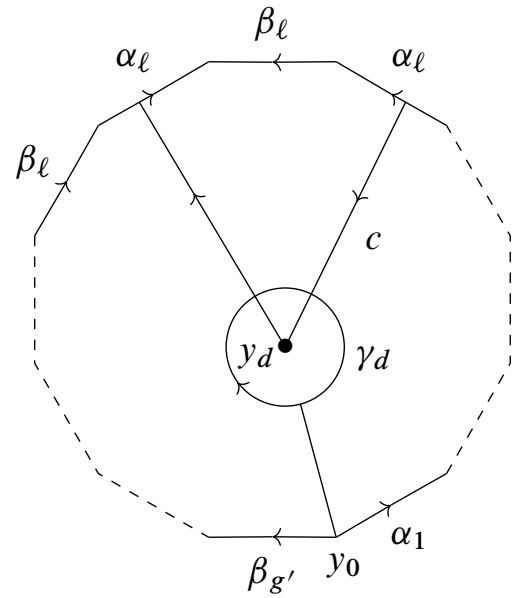

Figure 1.

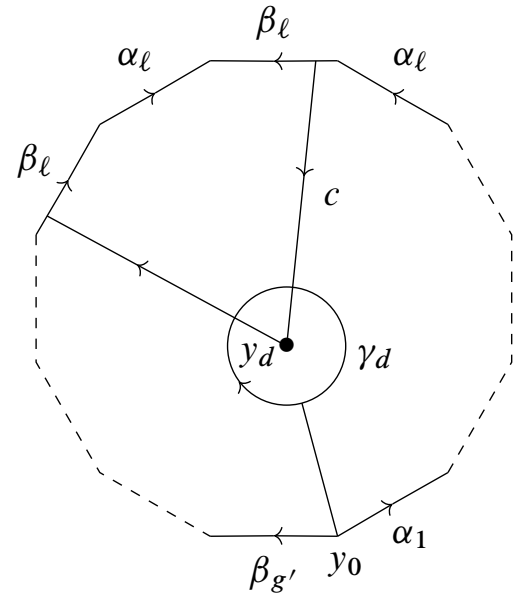

Figure 2.

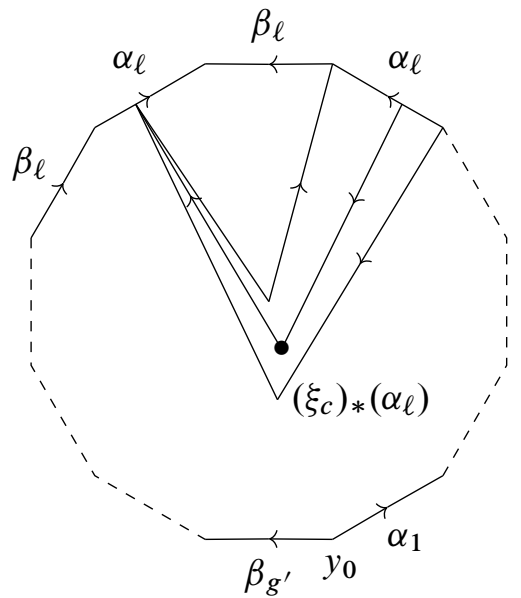

Figure 3. 
Proposition A.3. Let

$$
\Pi_{g^{\prime}}=\left\langle\alpha_{1}, \ldots, \beta_{g^{\prime}} \mid \prod_{1}^{g^{\prime}}\left[\alpha_{i}, \beta_{i}\right]\right\rangle
$$

and

$$
\Pi_{g^{\prime}-1}=\left\langle\alpha_{2}, \ldots, \beta_{g^{\prime}} \mid \prod_{2}^{g^{\prime}}\left[\alpha_{i}, \beta_{i}\right]\right\rangle .
$$

Then, for any $\varphi \in \operatorname{Aut}^{0}\left(\Pi_{g^{\prime}-1}\right)$, there exist $\psi \in \operatorname{Aut}^{0}\left(\Pi_{g^{\prime}}\right)$ and $\delta \in \Pi_{g^{\prime}}$ such that

$$
\begin{aligned}
& \psi\left(\alpha_{1}\right)=\alpha_{1}, \\
& \psi\left(\beta_{1}\right)=\beta_{1},
\end{aligned}
$$

and, for $i>1$,

$$
\begin{aligned}
& \psi\left(\alpha_{i}\right)=\delta \varphi\left(\alpha_{i}\right) \delta^{-1}, \\
& \psi\left(\beta_{i}\right)=\delta \varphi\left(\beta_{i}\right) \delta^{-1} .
\end{aligned}
$$

Proof. We first extend $\varphi$ to an automorphism

$$
\tilde{\varphi} \in \operatorname{Aut}\left(\left\langle\alpha_{2}, \ldots, \beta_{g^{\prime}}, \gamma \mid \gamma \cdot \prod_{2}^{g^{\prime}}\left[\alpha_{i}, \beta_{i}\right]\right\rangle\right)
$$

such that $\tilde{\varphi}\left(\alpha_{i}\right)=\varphi\left(\alpha_{i}\right), \tilde{\varphi}\left(\beta_{i}\right)=\varphi\left(\beta_{i}\right)$ and $\tilde{\varphi}(\gamma)=\delta^{-1} \gamma \delta, i>1$. Geometrically this corresponds to representing $\varphi$ as composition of Dehn twists along curves contained in the complement $Y_{g^{\prime}-1} \backslash D$ of a closed disk $D$ in a Riemann surface $Y_{g^{\prime}-1}$ of genus $g^{\prime}-1$, where $D$ does not intersect $\alpha_{i}$ and $\beta_{i}$.

Now simply define

$$
\begin{aligned}
& \psi\left(\alpha_{1}\right)=\alpha_{1}, \\
& \psi\left(\beta_{1}\right)=\beta_{1}, \\
& \psi\left(\alpha_{i}\right)=\delta \tilde{\varphi}\left(\alpha_{i}\right) \delta^{-1}, \\
& \psi\left(\beta_{i}\right)=\delta \tilde{\varphi}\left(\beta_{i}\right) \delta^{-1},
\end{aligned}
$$

for $i>1$. 


\section{B. Loci and topological types}

In this appendix we show that, up to essentially only one exception, the loci $M_{g, \rho}\left(D_{n}\right)$ are in bijection with the unmarked topological types of $D_{n}$-actions.

In general, thanks to the work of of Singerman, Ries, and Magaard-ShaskaShpectorov-Völklein (see [34], [32], and [30]), this is true more generally for the irreducible components of the loci $M_{g}(G)$; with a finite number of exceptions, they correspond bijectively to the unmarked topological types of $G$-actions. That there are indeed groups $G$ and different unmarked topological types of $G$-actions which yield the same component was already shown by Ries ([32]).

To have a simple notation, assume in this appendix that $H, H^{\prime}$ are distinct finite subgroups of the mapping class group $\mathrm{Map}_{g}$, and denote by

$$
Z:=\operatorname{Fix}(H), \quad Z^{\prime}:=\operatorname{Fix}\left(H^{\prime}\right)
$$

the corresponding irreducible analytic subsets of Teichmüller space $\mathcal{T}_{g}$. We already observed that, if we denote by

$$
\rho: H \longrightarrow \operatorname{Map}_{g}, \quad \rho^{\prime}: H^{\prime} \longrightarrow \operatorname{Map}_{g}
$$

the inclusion homomorphisms, these analytic subsets map to irreducible closed algebraic sets $M_{g, \rho}(H)$, respectively $M_{g, \rho^{\prime}}\left(H^{\prime}\right)$.

We define the generic group of automorphisms of a curve in $Z$ as

$$
G:=G_{H}:=\bigcap_{C \in Z} \operatorname{Stab}_{C} \quad\left(\operatorname{Aut}(C) \cong \operatorname{Stab}_{C} \subset \operatorname{Map}_{g}\right) .
$$

We refer to lemma 4.1 of [30] for the proof of the following result.

Theorem B.1. (MSSV) Suppose $H \subset G$ and $Z$ are as above, with $H$ a proper subgroup of $G$ and $C \in Z$. Then

$$
\delta:=\operatorname{dim}(Z) \leq 3 .
$$

I) if $\delta=3$, then $H$ has index 2 in $G$, and $C \rightarrow C / G$ is covering of $\mathbb{P}^{1}$ branched on six points, $P_{1}, \ldots, P_{6}$, and with branching indices all equal to 2 . Moreover the subgroup $H$ corresponds to the unique genus two double cover of $\mathbb{P}^{1}$ branched on the six points, $P_{1}, \ldots, P_{6}$ (by Galois theory, intermediate covers correspond to subgroups of $G$ bijectively).

II) If $\delta=2$, then $H$ has index 2 in $G$, and $C \rightarrow C / G$ is covering of $\mathbb{P}^{1}$ branched on five points, $P_{1}, \ldots, P_{5}$, and with branching indices $2,2,2,2, c_{5}$. Moreover the subgroup $H$ corresponds to a genus one double cover of $\mathbb{P}^{1}$ branched on four of the points $P_{1}, \ldots, P_{4}, P_{5}$ which have branching index 2 . 
III) If $\delta=1$, then there are three possibilities.

III-a) $H$ has index 2 in $G$, and $C \rightarrow C / G$ is covering of $\mathrm{P}^{1}$ branched on four points, $P_{1}, \ldots, P_{4}$, with branching indices $2,2,2,2 d_{4}$, where $d_{4}>1$. Moreover the subgroup $H$ corresponds to the unique genus one double cover of $\mathrm{P}^{1}$ branched on the four points, $P_{1}, \ldots, P_{4}$.

III-b) $H$ has index 2 in $G$, and $C \rightarrow C / G$ is covering of $\mathrm{P}^{1}$ branched on four points, $P_{1}, \ldots, P_{4}$, with branching indices $2,2, c_{3}, c_{4}$, where $c_{3} \leq c_{4}>2$. Moreover the subgroup $H$ corresponds to a genus zero double cover of $\mathrm{P}^{1}$ branched on two points whose branching index equals 2.

III-c) $H$ is normal in $G, G / H \cong(\mathbb{Z} / 2)^{2}$, moreover $C \rightarrow C / G$ is covering of $\mathrm{P}^{1}$ branched on four points, $P_{1}, \ldots, P_{4}$, with branching indices $2,2,2, c_{4}$, where $c_{4}>2$. Moreover the subgroup $H$ corresponds to the unique genus zero cover of $\mathbb{P}^{1}$ with group $(\mathbb{Z} / 2)^{2}$ branched on the three points $P_{1}, P_{2}, P_{3}$ whose branching index equals 2.

Corollary B.2. Assume that

$$
Z:=\operatorname{Fix}(H)=Z^{\prime}:=\operatorname{Fix}\left(H^{\prime}\right)
$$

where $H \neq H^{\prime}$ ( hence, in the previous notation, $H$ is a proper subgroup of $G$ ).

Then $\delta:=\operatorname{dim}(Z) \leq 2$. Moreover, if $\delta=2$, then necessarily all the five branching indices are equal to $2\left(c_{5}=2\right)$.

Assume further that $H, H^{\prime}$ have the same cardinality.

Then $G:=G_{H}:=\cap_{C \in Z} \operatorname{Stab}_{C}$ induces a sequence of coverings

$$
C \longrightarrow C / H \longrightarrow C / G
$$

which is not of type III-c), and, if it is type III-b), then the branching indices $2,2, c_{3}, c_{4}$ must satisfy $c_{3}=2$.

If $\mathrm{C} \rightarrow \mathrm{C} / \mathrm{H} \rightarrow \mathrm{C} / \mathrm{G}$ is of type III-a), then $\mathrm{C} \rightarrow \mathrm{C} / \mathrm{H}^{\prime} \rightarrow \mathrm{C} / \mathrm{G}$ is of type III-b) with branching indices $2,2,2,2 d_{4}$.

If moreover $\delta=0$, and $H \cong H^{\prime} \cong D_{n}$, then necessarily $Z$ and $Z^{\prime}$ are distinct points.

Proof. We can apply the previous theorem to both $H$ and $H^{\prime}$, which are proper subgroups of the same group $G$.

In the case I) where $\delta=3$, then both $H$ and $H^{\prime}$ correspond to the unique genus two cover branched on the six points, contradicting $H \neq H^{\prime}$.

The same argument applies in case II) when $c_{5}>2$. 
In the case where the covering $C \rightarrow C / H \rightarrow C / G$ is of type III-c), then the index of $H$ is four and, since $|H|=\left|H^{\prime}\right|$, the index of $H^{\prime}$ is also four, hence $H^{\prime}$ corresponds to the unique genus zero $(\mathbb{Z} / 2)^{2}$-cover branched on the three points $P_{1}, P_{2}, P_{3}$; again we obtain the contradiction $H=H^{\prime}$.

If the sequence of coverings $C \rightarrow C / H \rightarrow C / G$ is of type III-b), then the intermediate genus zero covering is unique unless $c_{3}=2$.

If the type is III-a), the type for $H^{\prime}$ cannot be the same, since we have a unique intermediate genus one cover. Since $\delta=1$, the index of $H^{\prime}$ is also equal to two, and the branching indices are 2,2,2,2 $d_{4}$ the type of $H^{\prime}$ must be III-b).

Finally, if $\delta=0$ and $H \cong H^{\prime} \cong D_{n}$, we have a dihedral covering of $\mathbb{P}^{1}$ branched in three points, for which there is a unique topological type (see [10]), corresponding to the monodromy factors $y, y x, x^{-1}$.

Remark B.3. In the case where $H \cong H^{\prime} \cong D_{n}$, and $H \neq H^{\prime}$ we have then $\delta=1$ or $\delta=2$. Moreover, both $H, H^{\prime}$ have index in $G$ equal to 2 .

If $\delta=2$, then $H, H^{\prime}$ are both of type II), hence they correspond to intermediate genus one covers branched on four of the five points $P_{1}, P_{2}, \ldots, P_{5}$.

If $\delta=1$, either $H, H^{\prime}$ are both of type III-b) (with branching indices 2, 2, 2, $c_{4}$ ) hence they correspond to intermediate genus zero covers branched on two of the three points $P_{1}, P_{2}, P_{3}$; or, up to exchanging $H$ with $H^{\prime}, H$ is of type III-a) and $H^{\prime}$ is of type III-b) with branching indices $2,2,2,2 d_{4}$.

The investigation and detailed classification of these coincidences $\left(Z=Z^{\prime}\right.$ and $H \cong H^{\prime} \cong D_{n}$, with $H \neq H^{\prime}$ ) is interesting, but shall not be fully pursued here. We shall limit ourselves to show that it must be $\delta=1$, and that there is only one exception, namely, the one where one group is of type III-a) and the other of type III-b).

A common feature of all the cases is however the following situation. Defining $K=H \cap H^{\prime}$, we have an exact sequence

$$
1 \longrightarrow K \longrightarrow G \longrightarrow(\mathbb{Z} / 2)^{2} \longrightarrow 1 .
$$

Proposition B.4. Assume that we have an exact sequence $(D D)$ where $H \cong H^{\prime} \cong$ $D_{n}$. Then there are elements $\gamma_{1}, \gamma_{2}$ with $\gamma_{1}^{2}=\gamma_{2}^{2}=1$, whose images generate $(\mathbb{Z} / 2)^{2}$, and such that

$$
H=\left\langle K, \gamma_{1}\right\rangle, H^{\prime}=\left\langle K, \gamma_{2}\right\rangle .
$$

Moreover $(D D)$ splits if and only if $G \cong D_{n} \times \mathbb{Z} / 2, H$ corresponds to the subgroup $D_{n} \times\{0\}$, and $H^{\prime}$ is the graph of a homomorphism $\phi: D_{n} \rightarrow \mathbb{Z} / 2$ with kernel equal to $K$. 
If $(D D)$ does not split, then $n$ is even and either $G \cong D_{2 n}$ or $n=4 h$, where $h$ is odd, and $G$ is the semidirect product of $H \cong D_{n}$ with $\left\langle\gamma_{2}\right\rangle \cong \mathbb{Z} / 2$, such that conjugation by $\gamma_{2}$ acts as follows:

$$
y \longmapsto y x^{2}, \quad x \longmapsto x^{2 h-1} .
$$

Remark B.5. The condition that (DD) splits always holds, except possibly if $H, H^{\prime}$ are of different types III-a) and III-b).

Indeed if $H, H^{\prime} \cong D_{n}$ are both of type II), then the Hurwitz generating systems of $C \rightarrow C / H, C \rightarrow C / H^{\prime}$ must be of the form

$$
v=\left(c_{1}, c_{2} ; a, b\right), \quad v^{\prime}=\left(c_{1}^{\prime}, c_{2}^{\prime} ; a^{\prime}, b^{\prime}\right) \in \operatorname{HS}\left(D_{n} ; 1,2\right),
$$

where $c_{2}$ is conjugate to $c_{1}$ (resp. $c_{2}^{\prime}$ is conjugate to $c_{1}^{\prime}$ ) in $G$ and $c_{i}^{2}=\left(c_{i}^{\prime}\right)^{2}=1$, $i=1$, 2. From Theorem B.1 it follows that $c_{1}, c_{2}, c_{1}^{\prime}, c_{2}^{\prime} \notin K=H \cap H^{\prime}$, therefore $c_{1}, c_{2}$ (resp. $c_{1}^{\prime}, c_{2}^{\prime}$ ) are reflections in $H$ (resp. $H^{\prime}$ ).

Since otherwise one of them must be the unique central element of $H$ (resp. of $H^{\prime}$ ); but the unique central element of $H$ is then central in $G$ ( $H$ being normal in $G$ ), hence it equals the unique central element of $H^{\prime}$ by the same argument: hence it lies in $K$ and we derive a contradiction.

From this we deduce that the numerical type of $v$ is the same as that of $v^{\prime}$, up to automorphisms, and hence using Proposition 5.4 it follows that $H$ and $H^{\prime}$ have the same unmarked topological type.

Assume that $H, H^{\prime} \cong D_{n}$ are both of type III-b). Let $v=\left(v_{1}, v_{2}, v_{3}, v_{4}\right)$ in $\operatorname{HS}(G ; 0,4)$ be the Hurwitz generating system of $C \rightarrow C / G$. By Corollary B.2 we have

$$
v_{1}^{2}=v_{2}^{2}=v_{3}^{2}=1 \neq v_{4}^{2} .
$$

Moreover, by Theorem B.1, $v_{4} \in H \cap H^{\prime}=K$. Since $v_{4}^{2} \neq 1, v_{4}$ is a rotation in $H$ and in $H^{\prime}$. Now consider the Hurwitz systems of $C \rightarrow C / H$ and of $C \rightarrow C / H^{\prime}$, which are of the following forms:

$$
u=\left(u_{1}, u_{2}, v_{4}, v_{4}^{\prime}\right), \quad u^{\prime}=\left(u_{1}^{\prime}, u_{2}^{\prime}, v_{4}, v_{4}^{\prime \prime}\right),
$$

where $v_{4}^{\prime}$ is conjugate to $v_{4}$ in $G$. Notice that each of them contains only two reflections, hence by Remark 5.2 (R) $v_{4}$ must generate the subgroup of rotations in $H$ (resp. in $H^{\prime}$ ). But $v_{4} \in K$ and the subgroup of rotations of $K$ have index 2 in the subgroup of rotations of $H$ (resp. of $H^{\prime}$ ) if $G$ is not the direct product of $D_{n}$ with $\mathbb{Z} / 2$. 
Proof of Proposition B.4. $K$ is an index two subgroup in $D_{n}$. If $n$ is odd, then necessarily $K=\mathcal{R}$, the subgroup of rotations.

1) More generally, if $K=\mathcal{R}$, any element in $H \backslash K$ has order two and splits the exact sequence $1 \rightarrow K \rightarrow H \rightarrow(\mathbb{Z} / 2) \rightarrow 1$, and the same holds for $H^{\prime}$. Therefore we find elements $\gamma_{1}, \gamma_{2}$ as desired, and such that their conjugation action on $K$ is the same, $x^{i} \mapsto x^{-i}$.

Now, $\gamma:=\gamma_{1} \gamma_{2}$ centralizes $K=\mathcal{R}$ and $\gamma^{2} \in K$. Let $x$ be generator of $\mathcal{R}$. Hence

$$
\gamma_{1} \gamma_{2} \gamma_{1} \gamma_{2}=x^{r} \text {. }
$$

Replacing $\gamma_{2}$ with $\gamma_{2} x^{a}$, we replace $r$ by $r+2 a$. If $n$ is odd, then we may assume $r=0$, whereas if $n$ is even, we also have the case $r=1$. Observe that $r=0$ implies the splitting of the above sequence (DD), moreover $\gamma$ centralizes $K$.

2) Assume that (DD) splits and $\gamma=\gamma_{1} \gamma_{2}$ centralizes $K$. Then

$$
\gamma_{1} \gamma_{2} \gamma_{1} \gamma_{2}=1 \longleftrightarrow \gamma \gamma_{1} \gamma=\gamma_{1},
$$

which amounts to $\gamma$ being in the centre of $G$. Hence

$$
G \cong H \times\langle\gamma\rangle \cong H \times \mathbb{Z} / 2 .
$$

Moreover, $H^{\prime}=\left\langle K, \gamma_{2}\right\rangle=\left\langle K, \gamma_{1} \gamma\right\rangle$ which proves our assertion.

3) Assume now that $K=\mathcal{R}$ and that (DD) does not split: then we can only achieve $\gamma^{2}=x$. Observe now that

$$
\gamma_{1} \gamma \gamma_{1}=\gamma_{2} \gamma_{1}=\gamma^{-1}, \gamma^{i}=1 \longleftrightarrow 2 n \mid i .
$$

Since $G$ is generated by $\gamma, \gamma_{1}$, it follows that $G \cong D_{4 m}, n=2 m$.

4) Assume now that $n=2 m$ is even and $K \neq \mathcal{R}$. Then $K \cong D_{m}$, and $K$ is generated, up to an automorphism, by $x^{2}$ and $y$. The element $z:=y x$ has order two and its action by conjugation is

$$
x^{2} \longmapsto x^{-2}, \quad y \longmapsto y x^{2} .
$$

In this way we construct also in this case the desired elements $\gamma_{1}, \gamma_{2}$ such that $\gamma:=\gamma_{1} \gamma_{2}$ centralizes $K$.

Now, $\gamma^{2}$ is in the centre of $D_{m}$, hence $\gamma^{2}=1$ if $m$ is odd, or, if $m=2 h$, then $\gamma^{2}=x^{2 h}=x^{m}$.

If $\gamma^{2}=1$ we have the splitting and we can apply 2).

Otherwise, if $h$ is even, replace $\gamma_{2}$ by $\gamma_{2} x^{h}$, which has again order equal to two. Then $\gamma$ is replaced by $\xi:=\gamma_{1} \gamma_{2} x^{h}$. $\xi$ has order two, since

$$
\xi^{2}=\gamma_{1} \gamma_{2} x^{h} \gamma_{1} \gamma_{2} x^{h}=\gamma_{1} \gamma_{2} \gamma_{1} \gamma_{2} x^{2 h}=\gamma^{2} x^{m}=x^{2 m}=1 .
$$


Observe then that $\gamma_{1} \gamma_{2}=\gamma_{2} \gamma_{1} x^{m}$.

We show that then $\xi$ is in the centre:

$$
\begin{gathered}
\gamma_{1} \xi \gamma_{1}:=\gamma_{1} \gamma_{1} \gamma_{2} x^{h} \gamma_{1}=\gamma_{2} \gamma_{1} x^{-h}=\gamma_{1} \gamma_{2} x^{-m-h}=\gamma_{1} \gamma_{2} x^{h}=\xi, \\
\gamma_{2} \xi \gamma_{2}:=\gamma_{2} \gamma_{1} \gamma_{2} x^{h} \gamma_{2}=\gamma_{2} \gamma_{2} \gamma_{1} x^{m+h} \gamma_{2}=\gamma_{1} x^{-h} \gamma_{2}=\gamma_{1} \gamma_{2} x^{h}=\xi .
\end{gathered}
$$

Assume instead that $h$ is odd. Then we observe simply that $G$ is generated by $H$ and by $\gamma_{2}$, and we set $x:=y \gamma_{1} \in H$, so that $H$ is generated in the standard way by $y, x$.

We have $\gamma_{2}^{2}=1$, moreover conjugation by $\gamma_{2}$ sends

$$
x^{2} \longmapsto x^{-2}, \quad y \longmapsto y x^{2}, \quad \gamma_{1} \longmapsto \gamma_{1} x^{2 h},
$$

since $\gamma_{1} \gamma_{2} \gamma_{1} \gamma_{2}=x^{2 h}$.

We conclude that

$$
x:=y \gamma_{1} \mapsto y x^{2} \gamma_{1} x^{2 h}=y x^{2} y x x^{2 h}=x^{2 h-1} .
$$

Proposition B.6. The case $\delta=2$ cannot occur for the group $D_{n}$.

Proof. We have already shown that in case II) the five branching indices must all be equal to 2 .

By B.4 and B.5 the group $G$ generated by $H, H^{\prime}$ must be $G=D_{n} \times C_{2}$, where $C_{2}:=\mathbb{Z} / 2, H$ is the subgroup $D_{n} \times\{0\}$, while $H^{\prime}$ is the graph of a homomorphism of $H$ onto $C_{2}$.

We consider first the case where four of the five elements have a reflexion component. To have $(y, 0)$ in the group they generate, the second component of one of these four elements must be trivial. We permute the other three into the first three positions, apply Lemma 2.1 of [10] to make the first two equal. So after a suitable automorphism of $D_{n}$ the tuple is

$$
(y, 1)(y, 1)\left(y x^{\ell}, 1\right)\left(y x^{\ell+m}, 0\right)\left(x^{m}, 1\right)
$$

where $m$ is an integer in $\left\{0, \frac{n}{2}\right\}$.

In case $n$ even and $m=n / 2$ we may apply the following automorphism of $G$, $(x, 0) \mapsto(x, 0),(y, 0) \mapsto\left(y x^{m}, 0\right),(e, 1) \mapsto\left(x^{m}, 1\right)$. 
So we are left with the case $m=0$, where we can apply another automorphism to get

$$
(y, 1)(y, 1)(y x, 1)(y x, 0)(e, 1) .
$$

Since only one of these elements is in $H^{\prime}$, and $H^{\prime} \neq H, H^{\prime}$ is the graph of the homomorphism which sends $y$ to 0 and $x$ to 1 , in particular $n$ must be even.

On the two intermediate covers, which are elliptic curves, we get the respective Hurwitz vectors $(y x, y x ; x, 1)$ and $\left(y x^{\prime}, y x^{\prime} ; x^{\prime}, 1\right)$, where $x^{\prime}$ is a shorthand for $(x, 1) \in H^{\prime}$. Obviously they are the same under the automorphism of $G$ which keeps $(y, 0)$ and $(y, 1)$ fixed and sends $(x, 0)$ to $(x, 1)$. This means that $H=H^{\prime}$, a contradiction.

The analysis in the second case, where two of the five elements have a reflection component, is similar. Up to automorphisms and braid equivalence, there is only one possible tuple and $n$ must equal 2:

$$
(x, 1)(0,1)(x, 1)(y, 1)(y, 0) .
$$

Here $H^{\prime}$ must be the graph of the homomorphism which sends $y$ to 1 and $x$ to 0 . And on the two intermediate covers we get the respective Hurwitz vectors $(y, y ; x, x)$ and $\left(y^{\prime}, y^{\prime} ; x, x\right)$, where $y^{\prime}$ is a shorthand for $(y, 1) \in H^{\prime}$. They are in one orbit under the automorphism of $G$ which keeps $(x, 0)$ and $(x, 1)$ fix and which exchanges $(y, 0)$ with $(y, 1)$.

Theorem B.7. Assume that we have two distinct subgroups of $\mathrm{Map}_{g}, H, H^{\prime} \cong D_{n}$, and that $Z:=\operatorname{Fix}(H)=\operatorname{Fix}\left(H^{\prime}\right)$. Then $\delta:=\operatorname{dim}(Z)=1$, and the numerical invariant $g^{\prime}$ cannot be the same for both actions.

Proof. We have shown in Corollary B. 2 that $\delta \leq 2, \delta \neq 0$, and in Proposition B.6 that $\delta \neq 2$. Hence $\delta=1$.

By Remark B.3, if we make the assumption that $g^{\prime}$ is the same, follows then that $H$ and $H^{\prime}$ are both of type III-b). By Proposition B.4 and Remark B.5, the group is then $G=D_{n} \times C_{2}$,

$H$ is $D_{n} \times\{0\}$ and $H^{\prime}$ is the graph of some homomorphism from $D_{n}$ to $C_{2}$. By the last paragraph of Remark B.5, $K$ coincides with the group of rotations of $H=D_{n} \times\{0\}$, hence $H^{\prime}=\operatorname{ker}(f)$, where

$$
f: G=D_{n} \times C_{2} \rightarrow C_{2}
$$

is given by

$$
f\left(y^{b} x^{i}, a\right)=a+b .
$$


By Remark B.3, the branching indices of $C \rightarrow C / G$ are 2,2,2, $c_{4}$, therefore up to a permutation we get the sequences

$$
a=1,1,0,0, \quad a+b=0,1,1,0, \quad b=1,0,1,0 .
$$

Hence we get that the first coordinates of the second and of the last element are rotations of respective orders $d_{2}, c_{4}$, where $d_{2}=1$ or $d_{2}=2$.

There is surjection $G \rightarrow D_{n}$ with the second element in the kernel. Now, if $D_{n}$ is generated by two reflections $\sigma_{1}, \sigma_{2}$ then the rotation $\sigma_{1} \sigma_{2}$ generates the group $\mathcal{R}$ of rotations. We conclude that the order $c_{4}=n$.

We may assume, up to an automorphism of $D_{n}$, that the last element is $(x, 0)$, and the first one is $(y, 1)$.

Then the 4-tuple is

$$
(y, 1)\left(x^{h}, 1\right)\left(y x^{h-1}, 0\right)(x, 0),
$$

where $h=0$ or $h=n / 2$.

The respective subgroups are generated by $\gamma_{3}, \gamma_{4}, \gamma_{1} \gamma_{3} \gamma_{1}=: \gamma_{3}^{\prime}, \gamma_{1} \gamma_{4} \gamma_{1}=: \gamma_{4}^{\prime}$ in one case, by $\gamma_{4}, \gamma_{1}^{*}:=\gamma_{1}^{-1}, \gamma_{2} \gamma_{4} \gamma_{2}, \gamma_{2} \gamma_{1}^{*} \gamma_{2}$ in the other.

We get the corresponding length four Hurwitz vectors,

$$
\left(y x^{h-1}, x, y x^{h+1}, x^{-1}\right), \quad(x, y, x, y) .
$$

The corresponding two Nielsen functions are equal for $n$ odd $(h=0)$, distinct for $n$ even, but in the same orbit under the group $\operatorname{Aut}\left(D_{n}\right)$.

By [10], Theorem 2, $H, H^{\prime}$ correspond then to the same unmarked topological type.

Theorem B.8. Assume that we have two distinct subgroups of $\mathrm{Map}_{g}, H, H^{\prime} \cong D_{n}$, and that $Z:=\operatorname{Fix}(H)=\operatorname{Fix}\left(H^{\prime}\right)$. Then $\delta:=\operatorname{dim}(Z)=1$, and case III-a) holds for $H$, case III-b) holds for $H^{\prime}$.

This case actually occurs.

Proof. That the only possible case is the one described follows by theorem B.7 and the previous discussion. It suffices thus to give a concrete example.

We have a polygonal group $T(2,2,2,2 d)$, generated by elements $\gamma_{1}, \gamma_{2}, \gamma_{3}$, $\gamma_{4}$ whose orders are respectively 2,2,2,2d and whose product $\gamma_{1} \gamma_{2} \gamma_{3} \gamma_{4}$ is the identity. 
The elliptic induced covering is generated by

$$
a:=\gamma_{1} \gamma_{2}, \quad b:=\gamma_{2} \gamma_{3}, \quad c:=\left(\gamma_{1} \gamma_{4} \gamma_{1}\right)^{2}
$$

which satisfy the usual presentation $[a, b]=c$.

Whereas for the genus zero induced covering, since we have a double cover branched on the first two points, we get generators

$$
\gamma_{3}, \quad \gamma_{4}, \quad \gamma_{3}^{\prime}:=\gamma_{1} \gamma_{3} \gamma_{1}, \quad \gamma_{4}^{\prime}:=\gamma_{1} \gamma_{4} \gamma_{1},
$$

which satisfy the usual presentation

$$
\gamma_{3} \gamma_{4} \gamma_{3}^{\prime} \gamma_{4}^{\prime}=1
$$

These elements have respective orders $2,2 d, 2,2 d$, so we guess that $\gamma_{4}$ should map to a generator of the rotation group, say $\gamma_{4} \mapsto x$, and $\gamma_{3}$ should map to a reflection, say $\gamma_{3} \mapsto y$.

We make the following assumption: since $\gamma_{1}$ normalizes $H^{\prime}$, let us just assume that $\gamma_{1}$ centralizes $H^{\prime}$.

Then we have a direct product $G=H^{\prime} \times C_{2} \cong D_{n} \times C_{2}$, where $n=2 d$, and the cyclic group $C_{2}$ of order 2is generated by $\gamma_{1}$.

We take as Hurwitz vector for $G$ (images of $\gamma_{1}, \gamma_{2}, \gamma_{3}, \gamma_{4}$ )

$$
(0,1)(y x, 1)(y, 0)(x, 0) \text {. }
$$

Clearly these four elements generate then $G$.

Now $\gamma_{3}, \gamma_{4}, \gamma_{3}^{\prime}, \gamma_{4}^{\prime}$ are respectively sent to $(y, 0)(x, 0)(y, 0)(x, 0)$, and they generate the group $H^{\prime} \cong D_{n}$.

On the other hand

$$
a \longmapsto(y x, 0), \quad b \longmapsto\left(x^{-1}, 1\right), \quad c \longmapsto\left(x^{2}, 0\right)
$$

and clearly $[a, b] \mapsto\left(x^{2}, 0\right)$.

Moreover, the image group $H$ projects, under the first coordinate, onto the dihedral group $D_{n}$.

Acknowledgement. The authors would like to thank Binru Li and Sascha Weigl for providing the argument which helped us to fix a gap in a previous proof of Proposition B.6. 


\section{References}

[1] E. Artin, Geometric algebra. Interscience tracts in pure and applied mathematics, 3. Interscience Publishers, New York and London, 1957. Zbl 0077.02101 MR 0082463

[2] I. Bauer and F. Catanese, Generic lemniscates of algebraic functions. Math. Ann. 307 (1997), no. 3, 417-444. Zbl 0873.57004 MR 1437047

[3] R. Biggers and M. Fried, Irreducibility of moduli spaces of cyclic unramified covers of genus $g$ curves. Trans. Amer. Math. Soc. 295 (1986), no. 1, 59-70. Zbl 0601.14022 MR 0831188

[4] J. S. Birman, Mapping class groups and their relationship to braid groups. Comm. Pure Appl. Math. 22 (1969), 213-238. Zbl 0167.21503 MR 0243519

[5] K. S. Brown, Cohomology of groups. Graduate Texts in Mathematics, 87. Springer, New York and Berlin, 1982. Zbl 0584.20036 MR 0672956

[6] F. Catanese, Moduli of algebraic surfaces. In E. Sernesi (ed.), Theory of moduli (Montecatini Terme, 1985). Lecture Notes in Mathematics, 1337. Springer, Berlin etc., 1988. Zbl 0658.14017 MR 0963062

[7] F. Catanese, Fibred surfaces, varieties isogenous to a product and related moduli spaces. Amer. J. Math. 122 (2000), no. 1, 1-44. Zbl 0983.14013 MR 1737256

[8] F. Catanese, Differentiable and deformation type of algebraic surfaces, real and symplectic structures. In F. Catanese and G. Tian (eds.), Symplectic 4-manifolds and algebraic surfaces (CIME, Cetraro, 2003). Lecture Notes in Mathematics, 1938. Springer, Berlin etc., and Fondazione CIME., Florence, 2008, 55-167. Zbl 2441412 MR 1145.14001

[9] F. Catanese, Irreducibility of the space of cyclic covers of algebraic curves of fixed numerical type and the irreducible components of Sing $\left(\overline{\mathfrak{M}_{g}}\right)$. In S. Janeczko, J. Li, and D. H. Phong (eds.), Advances in geometric analysis (Warsaw, 2009). Advanced Lectures in Mathematics (ALM), 21. International Press, Somerville, MA, and Higher Education Press, Beijing, 2012, 281-306. Zbl 1317.14058 MR 3077261

[10] F. Catanese, M. Lönne, and F. Perroni, Irreducibility of the space of dihedral covers of the projective line of a given numerical type. Atti Accad. Naz. Lincei Cl. Sci. Fis. Mat. Natur. Rend. Lincei (9) Mat. Appl. 22 (2011), no. 3, 291-309. Zbl 1268.14025 MR 2847474

[11] F. Catanese, M. Lönne, and F. Perroni, Genus stabilization for moduli of curves with symmetries. arXiv:1301.4409 [math.AG] To appear in Alg. Geom.

[12] A. Clebsch, Zur Theorie der Riemann'schen Flächen. Math. Ann. 6 (1873), 216-230. JFM 05.0285.03

[13] A. Comessatti, Sulle superficie multiple cicliche. Rendiconti Seminario Padova 1 (1930), 1-45. JFM 56.0321.03

[14] M. Cornalba, On the locus of curves with automorphisms. Ann. Mat. Pura Appl. (4) 149 (1987), 135-151. Zbl 0649.14013 MR 0932781 
[15] M. Cornalba, Erratum: On the locus of curves with automorphisms. Ann. Mat. Pura Appl. (4) 187 (2008), no. 1, 185-186. Zbl 1150.14003 MR 2346015

[16] P. Deligne and D. Mumford, The irreducibility of the space of curves of given genus. Inst. Hautes Études Sci. Publ. Math. 36 (1969), 75-109. Zbl 0181.48803 MR 0262240

[17] N. M. Dunfield and W. P. Thurston, Finite covers of random 3-manifolds. Invent. Math. 166 (2006), no. 3, 457-521. Zbl 1111.57013 MR 2257389

[18] A. L. Edmonds, Surface symmetry. I. Michigan Math. J. 29 (1982), no. 2, 171-183. Zbl 0511.57025 MR 0654478

[19] A. L. Edmonds, Surface symmetry. II. Michigan Math. J. 30 (1983), no. 2, 143-154. Zbl 0562.57016 MR 0718259

[20] B. Farb and D. Margalit, A primer on mapping class groups. volume 49 of Princeton Mathematical Series, Princeton Mathematical Series, 49. Princeton University Press, Princeton, N.J., 2012. Zbl 1245.57002 MR 2850125

[21] M. D. Fried and H. Völklein, The inverse Galois problem and rational points on moduli spaces. Math. Ann. 290 (1991), no. 4, 771-800. Zbl 0763.12004 MR 1119950

[22] W. Fulton, Hurwitz schemes and irreducibility of moduli of algebraic curves. Ann. of Math. (2) 90 (1969), 542-575. Zbl 0194.21901 MR 0260752

[23] T. Graber, J. Harris, and J. Starr, A note on Hurwitz schemes of covers of a positive genus curve. Preprint 2002. arXiv:math.AG/0205056 [math.AG]

[24] H. Hopf, Fundamentalgruppe und zweite Bettische Gruppe. Comment. Math. Helv. 14 (1942). 257-309. Zbl 0027.09503 JFM 68.0503.01 MR 0006510

[25] A. Hurwitz, Ueber Riemann'schen Flächen mit gegebenen Verzweigungspunkten. Math. Ann. 39 (1891), 1-61.

[26] V. Kanev, Hurwitz spaces of Galois coverings of $\mathbf{P}^{1}$, whose Galois groups are Weyl groups. J. Algebra 305 (2006), no. 1, 442-456. Zbl 1118.14034 MR 2264138

[27] V. Kanev, Irreducibility of Hurwitz spaces. arXiv:math.AG/0509154 [math.AG]

[28] P. Kluitmann, Hurwitz action and finite quotients of braid groups. In J. S. Birman and A. Libgober (eds.), Braids (Santa Cruz, CA, 1986). Contemporary Mathematics, 78. American Mathematical Society, Providence, R.I., 1988, 299-325. Zbl 0975086 MR 0701.20019

[29] C. Livingston, Stabilizing surface symmetries. Michigan Math. J. 32 (1985), no. 2, 249-255. Zbl 0586.57021 MR 0783579

[30] K. Magaard, T. Shaska, S. Shpectorov, and H. Völklein, The locus of curves with prescribed automorphism group. Sürikaisekikenkyūsho Kōkyūroku 1267 (2002), 112-141. Communications in arithmetic fundamental groups (Kyoto, 1999/2001). MR 1954371

[31] R. Pardini, Abelian covers of algebraic varieties. J. Reine Angew. Math. 417 (1991), 191-213. Zbl 0721.14009 MR 1103912

[32] J. F. X. Ries, Subvarieties of moduli space determined by finite groups acting on surfaces. Trans. Amer. Math. Soc. 335 (1993), no. 1, 385-406. Zbl 0784.32017 MR 1097170 
[33] C. Sia, Hurwitz equivalence in tuples of dihedral groups, dicyclic groups, and semidihedral groups. Electron. J. Combin. 16 (2009), no. 1, Research Paper 95, 17 pp. Zbl 1191.20035 MR 2529804

[34] D. Singerman, Finitely maximal Fuchsian groups. J. London Math. Soc. (2) 6 (1972), 29-38. Zbl 0251.20052 MR 0322165

[35] F. Vetro, Irreducibility of Hurwitz spaces of coverings with one special fiber. Indag. Math. (N.S.) 17 (2006), no. 1, 115-127. Zbl 1101.14040 MR 2337168

[36] F. Vetro, Irreducibility of Hurwitz spaces of coverings with monodromy groups Weyl groups of type $W\left(B_{d}\right)$. Boll. Unione Mat. Ital. Sez. B Artic. Ric. Mat. (8) 10 (2007), no. 2, 405-431. Zbl 1178.14029 MR 2339450

[37] F. Vetro, Irreducibility of Hurwitz spaces of coverings with one special fiber and monodromy group a Weyl group of type $D_{d}$. Manuscripta Math. 125 (2008), no. 3, 353-368. Zbl 1139.14023 MR 2373066

[38] B. Wajnryb, Orbits of Hurwitz action for coverings of a sphere with two special fibers. Indag. Math. (N.S.) 7 (1996), no. 4, 549-558. Zbl 0881.57001 MR 1620132

[39] B. Wajnryb, An elementary approach to the mapping class group of a surface. Geom. Topol. 3 (1999), 405-466. Zbl 0947.57015 MR 1726532

[40] J. Wiegold, The Schur multiplier: an elementary approach. In C. M. Campbell and E. F. Robertson (eds.), Groups - St. Andrews 1981. London Mathematical Society Lecture Note Series, 71. Cambridge University Press, Cambridge and New York, 1982, 137-154. Zbl 0502.20003 MR 0679156

Received February 9, 2014

Fabrizio Catanese, Lehrstuhl Mathematik VIII,

Mathematisches Institut der Universität Bayreuth, NW II, Universitätsstr. 30, 95447 Bayreuth, Deutschland

e-mail: fabrizio.catanese@uni-bayreuth.de

Michael Lönne, Lehrstuhl Mathematik VIII,

Mathematisches Institut der Universität Bayreuth, NW II, Universitätsstr. 30,

95447 Bayreuth, Deutschland

e-mail: michael.loenne@uni-bayreuth.de

Fabio Perroni, Dipartimento di Matematica e Geoscienze,

Università degli Studi di Trieste, Piazzale Europa 1, 34127 Trieste, Italia

e-mail: fperroni@units.it 